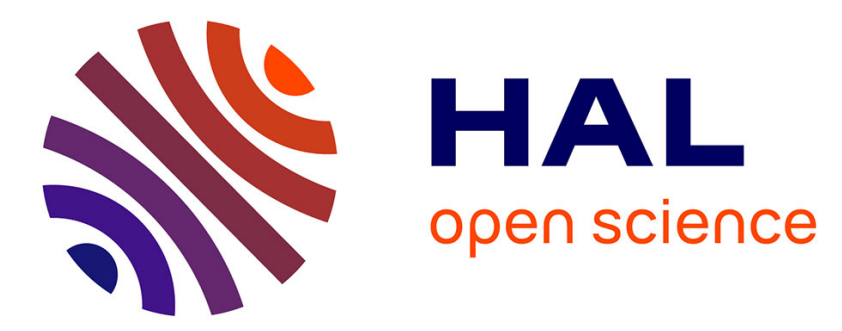

\title{
A modified Lagrange-Galerkin method for a fluid-rigid system with discontinuous density
}

\author{
Jorge San Martin, Jean-François Scheid, Loredana Smaranda
}

\section{To cite this version:}

Jorge San Martin, Jean-François Scheid, Loredana Smaranda. A modified Lagrange-Galerkin method for a fluid-rigid system with discontinuous density. Numerische Mathematik, 2012, 122 (2), pp.341-382. 10.1007/s00211-012-0460-1 . hal-00531433

\section{HAL Id: hal-00531433 \\ https://hal.science/hal-00531433}

Submitted on 2 Nov 2010

HAL is a multi-disciplinary open access archive for the deposit and dissemination of scientific research documents, whether they are published or not. The documents may come from teaching and research institutions in France or abroad, or from public or private research centers.
L'archive ouverte pluridisciplinaire HAL, est destinée au dépôt et à la diffusion de documents scientifiques de niveau recherche, publiés ou non, émanant des établissements d'enseignement et de recherche français ou étrangers, des laboratoires publics ou privés. 


\title{
A modified Lagrange-Galerkin method for a fluid-rigid system with discontinuous density
}

\author{
Jorge San Martín*, Jean-François Scheid ${ }^{\dagger}, \quad$ Loredana Smaranda ${ }^{\ddagger}$
}

\begin{abstract}
In this paper, we propose a new characteristics method for the discretization of the two dimensional fluid-rigid body problem in the case where the densities of the fluid and the solid are different. The method is based on a global weak formulation involving only terms defined on the whole fluid-rigid domain. To take into account the material derivative, we construct a special characteristic function which maps the approximate rigid body at the discrete time level $t_{k+1}$ into the approximate rigid body at time $t_{k}$. Convergence results are proved for both semi-discrete and fully-discrete schemes.
\end{abstract}

\section{Introduction}

The aim of this paper is to present a modified characteristics method for the discretization of the equations modelling the motion of a rigid solid immersed into a viscous incompressible fluid. Our method is a generalisation of the numerical scheme presented in San Martín, Scheid, Takahashi and Tucsnak [18, for the case where the fluid and the solid have different densities. The fluid-rigid system occupies a bounded and regular domain $\mathcal{O} \subset \mathbb{R}^{2}$. The solid is assumed to be a ball of radius 1 whose center, at time $t$, is denoted by $\boldsymbol{\zeta}(t)$. The fluid fills the part $\Omega(t)=\mathcal{O} \backslash B(\boldsymbol{\zeta}(t))$ at time $t$. The velocity field $\mathbf{u}(\mathbf{x}, t)$ and the pressure $p(\mathbf{x}, t)$ of the fluid, the center of mass $\zeta(t)$ and the angular velocity $\omega(t)$ of the ball satisfy the following Navier-Stokes system coupled with Newton's laws:

$$
\begin{aligned}
& \rho_{f}\left(\frac{\partial \mathbf{u}}{\partial t}+(\mathbf{u} \cdot \nabla) \mathbf{u}\right)-\mu \Delta \mathbf{u}+\nabla p=\rho_{f} \mathbf{f}, \mathbf{x} \in \Omega(t), t \in[0, T], \\
& \operatorname{div} \mathbf{u}=0, \mathbf{x} \in \Omega(t), t \in[0, T] \\
& \mathbf{u}=0, \mathbf{x} \in \partial \mathcal{O}, t \in[0, T], \\
& \mathbf{u}=\boldsymbol{\zeta}^{\prime}(t)+\omega(t)(\mathbf{x}-\boldsymbol{\zeta}(t))^{\perp}, \mathbf{x} \in \partial B(\boldsymbol{\zeta}(t)), t \in[0, T], \\
& m \boldsymbol{\zeta}^{\prime \prime}(t)=-\int_{\partial B(\boldsymbol{\zeta}(t))} \boldsymbol{\sigma} \mathbf{n} d \Gamma+\rho_{s} \int_{B(\boldsymbol{\zeta}(t))} \mathbf{f}(\mathbf{x}, t) d \mathbf{x}, t \in[0, T], \\
& J \omega^{\prime}(t)=-\int_{\partial B(\boldsymbol{\zeta}(t))}(\mathbf{x}-\boldsymbol{\zeta}(t))^{\perp} \cdot \boldsymbol{\sigma} \mathbf{n} d \Gamma+\rho_{s} \int_{B(\boldsymbol{\zeta}(t))}(\mathbf{x}-\boldsymbol{\zeta}(t))^{\perp} \cdot \mathbf{f}(\mathbf{x}, t) d \mathbf{x}, t \in[0, T]
\end{aligned}
$$

In the above system, $\boldsymbol{\sigma}=-p \mathbf{I d}+2 \mu \mathrm{D}(\mathbf{u})$ denotes the Cauchy stress tensor with $\mathrm{D}(\mathbf{u})=$ $\left(\nabla \mathbf{u}+\nabla \mathbf{u}^{T}\right) / 2$ and $\nabla \mathbf{u}^{T}$ means the transpose of $\nabla \mathbf{u}$. The positive constant $\mu$ is the dynamic viscosity of the fluid and the constants $m$ and $J$ are the mass and the moment of inertia

*Departamento de Ingeniería Matemática, Facultad de Ciencias Físicas y Matemáticas, Universidad de Chile and Centro de Modelamiento Matemático, UMR 2071 CNRS-UChile, Casilla 170/3-Correo 3, Santiago, Chile; jorge@dim.uchile.cl

${ }^{\dagger}$ Institut Elie Cartan UMR 7502, Nancy-Université, CNRS, INRIA, B.P. 239, F-54506 Vandoeuvre-lès-Nancy Cedex, France; scheid@iecn.u-nancy.fr

${ }^{\ddagger}$ Department of Mathematics, Faculty of Mathematics and Computer Science, University of Piteşti, Str. Târgu din Vale nr. 1, 110040 Piteşti, Romania; smaranda@dim.uchile.cl 
of the rigid body. Throughout this article, we will use the notation $\mathbf{x}^{\perp}=\left(\begin{array}{c}-x_{2} \\ x_{1}\end{array}\right)$ for all $\mathbf{x}=\left(\begin{array}{l}x_{1} \\ x_{2}\end{array}\right) \in \mathbb{R}^{2}$. System $1.1-1.6$ is completed with initial conditions:

$$
\begin{gathered}
\mathbf{u}(\mathbf{x}, 0)=\mathbf{u}_{0}(\mathbf{x}), \quad \mathbf{x} \in \Omega(0), \\
\boldsymbol{\zeta}(0)=\boldsymbol{\zeta}_{0} \in \mathbb{R}^{2}, \quad \boldsymbol{\zeta}^{\prime}(0)=\boldsymbol{\zeta}_{1} \in \mathbb{R}^{2}, \quad \omega(0)=\omega_{0} \in \mathbb{R} .
\end{gathered}
$$

In this paper, we suppose that the density $\rho_{f}$ of the fluid and the density $\rho_{s}$ of the solid are constant, but not equal, that is

$$
\rho_{f} \neq \rho_{s} .
$$

The fluid-structure interaction problem (1.1) $-(1.8)$ is characterized by the strong coupling between the nonlinear equations of the fluid and those of the structure, as well as the fact that the equations of the fluid are written in a variable domain in time, which depends on the displacement of the structure. From the numerical point of view, in this kind of problems it is necessary to solve equations on moving domains. For this reason, in recent years various authors have proposed a number of different techniques, some of which are the level set method (see Osher and Sethian [13]), the fictitious domain method (see Glowinski, Pan, Hesla, Joseph and Périaux [7, 8]), the immersed boundary method (see Peskin [14]) and the Arbitrary Lagrangian Eulerian (ALE) method (see Formaggia and Nobile [4], Gastaldi [5], Maury [11], Maury and Glowinski [12]).

In the sequel, we briefly recall some reference about the numerical convergence for NavierStokes equations, when the domain is independent of time. The Lagrange-Galerkin method has been proposed for the numerical treatment of convection-dominated equations and it is based on combining a Galerkin finite element procedure with a special discretisation of the material derivative along trajectories. Pironneau in [15] has given a detailed analysis of the method for the Navier-Stokes equations and Süli [21] has proved optimal error estimates for the Lagrange-Galerkin mixed finite element approximation of Navier-Stokes equations in a velocity/pressure formulation. We also mention the work of Achdou and Guermond [1, where convergence analysis of a finite element projection/Lagrange-Galerkin method for the incompressible Navier-Stokes equations is done.

The numerical analysis of some time decoupling algorithms for the simulation of the interaction between a fluid and a structure in the case where the deformation of the structure induces an evolution in the fluid domain has been developed by Grandmont, Guimet and Maday [9] (one dimensional problem). For the ALE method, the numerical analysis of the unsteady Stokes equations in a time dependent domain when the motion of the domain is given has been studied in San Martín, Smaranda and Takahashi [20. Moreover, Legendre and Takahashi [10] have combined the method of characteristics with a finite element approximation to derive error estimates in the ALE formulation of a two-dimensional problem describing the motion of a rigid body in a viscous fluid. In San Martín, Scheid, Takahashi and Tucsnak [17, 18, the authors have proved the convergence of a numerical method based on finite elements with a fixed mesh for a two dimensional fluid-rigid body problem with the densities of the fluid and the solid equal, i.e. $\rho_{f}=\rho_{s}$. Their numerical scheme is based on a standard characteristic function resulting from the classical formulation of the material derivative in the Navier-Stokes equations. The method introduced in 18 cannot be easily extended to our case $\rho_{f} \neq \rho_{s}$ where the global density is discontinuous by using the same characteristic function. In this paper, we introduce crucial modifications on the characteristic function, and we propose a new numerical scheme in order to prove a similar convergence result as in 18 . We think that this modification on the characteristic function should be useful to obtain convergent algorithms for the simulation of aquatic organisms in two and three dimensional cases (see San Martín, Scheid, Takahashi and Tucsnak [19]).

The paper is organized as follows. In the next section we introduce some notation and the functional spaces we work on. In Section 3 we discretize the fluid-structure interaction problem (1.1)-11.8 in time variable and we state our first main result given in Theorem 3.3 which consists in the convergence of the semi-discretization scheme. Section 4 is dedicated to the fully discretization in time and space variables and then we state our second main result 
given in Theorem 4.1 which concerns an error estimate for the fully-discrete formulation. Section 5 is devoted to some crucial properties on the characteristic functions associated with our schemes. The last two sections are focused on the proofs of the convergence results for both semi-discrete and fully-discrete formulations.

\section{Notation and functional spaces}

Throughout this paper, we shall use the classical Sobolev spaces $H^{s}(\mathcal{O}), H_{0}^{s}(\mathcal{O}), H^{-s}(\mathcal{O})$, $s \geqslant 0$ and the space of Lipschitz continuous functions $C^{0,1}(\overline{\mathcal{O}})$ on the closure of $\mathcal{O}$. We also define

$$
L_{0}^{2}(\mathcal{O})=\left\{f \in L^{2}(\mathcal{O}) \mid \int_{\mathcal{O}} f \mathrm{~d} \mathbf{x}=0\right\} .
$$

The usual inner product in $L^{2}(\mathcal{O})^{2}$ will be denoted by

$$
(\mathbf{u}, \mathbf{v})=\int_{\mathcal{O}} \mathbf{u} \cdot \mathbf{v} \mathrm{d} \mathbf{x} \quad \forall \mathbf{u}, \mathbf{v} \in L^{2}(\mathcal{O})^{2} .
$$

If $\mathbf{A}$ is a matrix, we denote by $\mathbf{A}^{T}$ its transpose. For any $2 \times 2$ matrices $\mathbf{A}, \mathbf{B} \in \mathcal{M}_{2 \times 2}$, we denote by $\mathbf{A}: \mathbf{B}$ their inner product $\mathbf{A}: \mathbf{B}=\operatorname{Trace}\left(\mathbf{A}^{T} \mathbf{B}\right)$, and by $|\mathbf{A}|$ the corresponding norm. For convenience, we use the same notation as in $(2.1)$ for the inner product in $L^{2}\left(\mathcal{O}, \mathcal{M}_{2 \times 2}\right)$, that is

$$
(\mathbf{A}, \mathbf{B})=\int_{\mathcal{O}} \mathbf{A}: \mathbf{B} \mathrm{d} \mathbf{x} \quad \forall \mathbf{A}, \mathbf{B} \in L^{2}\left(\mathcal{O}, \mathcal{M}_{2 \times 2}\right) .
$$

For $\boldsymbol{\zeta} \in \mathcal{O}$, we introduce the space of rigid functions in $B(\boldsymbol{\zeta})=\left\{\mathbf{x} \in \mathbb{R}^{2}:|\mathbf{x}-\boldsymbol{\zeta}| \leq 1\right\}$,

$$
\mathcal{K}(\boldsymbol{\zeta})=\left\{\mathbf{u} \in H_{0}^{1}(\mathcal{O})^{2} \mid \mathrm{D}(\mathbf{u})=0 \text { in } B(\boldsymbol{\zeta})\right\},
$$

the space of rigid functions in $B(\boldsymbol{\zeta})$ with divergence free in the whole domain $\mathcal{O}$,

$$
\widehat{\mathcal{K}}(\boldsymbol{\zeta})=\{\mathbf{u} \in \mathcal{K}(\boldsymbol{\zeta}) \mid \operatorname{div} \mathbf{u}=0 \text { in } \mathcal{O}\},
$$

and the space of the pressure

$$
M(\boldsymbol{\zeta})=\left\{p \in L_{0}^{2}(\mathcal{O}) \mid p=0 \text { in } B(\boldsymbol{\zeta})\right\} .
$$

Remark 2.1. For convenience, in the reminder of the paper, any velocity field in $\mathcal{K}(\boldsymbol{\zeta})$ will be extended by zero outside of $\mathcal{O}$.

According to Lemma 1.1 of [22, pp.18], for any $\mathbf{u} \in \mathcal{K}(\boldsymbol{\zeta})$, there exist $\mathbf{l}_{u} \in \mathbb{R}^{2}$ and $\omega_{\mathbf{u}} \in \mathbb{R}$ such that

$$
\mathbf{u}(\mathbf{y})=\mathbf{l}_{\mathbf{u}}+\omega_{\mathbf{u}}(\mathbf{y}-\boldsymbol{\zeta})^{\perp} \quad \forall \mathbf{y} \in B(\boldsymbol{\zeta}) .
$$

In addition, we define the density $\rho$ by the following piecewise constant function

$$
\rho(\mathbf{x})= \begin{cases}\rho_{s} & \text { if } \mathbf{x} \in B(\boldsymbol{\zeta}), \\ \rho_{f} & \text { if } \mathbf{x} \in \mathcal{O} \backslash B(\boldsymbol{\zeta}) .\end{cases}
$$

We notice that, by using the above definitions, for any $\mathbf{u}, \mathbf{v} \in \mathcal{K}(\boldsymbol{\zeta})$ we have

$$
(\rho \mathbf{u}, \mathbf{v})=\int_{\mathcal{O} \backslash B(\boldsymbol{\zeta})} \rho_{f} \mathbf{u} \cdot \mathbf{v} \mathrm{d} \mathbf{x}+M \mathbf{l}_{\mathbf{u}} \cdot \mathbf{l}_{\mathbf{v}}+J \omega_{\mathbf{u}} \omega_{\mathbf{v}} .
$$

The spaces 2.2 -2.3 are specific to our problem. In fact, if the solution $\mathbf{u}$ of $1.1-1.8$ is extended by

$$
\mathbf{u}(\mathbf{x}, t)=\boldsymbol{\zeta}^{\prime}(t)+\omega(t)(\mathbf{x}-\boldsymbol{\zeta}(t))^{\perp} \quad \forall \mathbf{x} \in B(\boldsymbol{\zeta}(t)),
$$

then, we easily see that $\mathbf{u}(t) \in \widehat{\mathcal{K}}(\boldsymbol{\zeta}(t))$. In the reminder of this paper, the solution $\mathbf{u}$ of (1.1) - 1.8) will be extended as above. 
An important ingredient of the numerical method we use is given by the characteristic functions whose level lines are the integral curves of the velocity field. More precisely (see, for instance, [15], [21]) the characteristic function $\widetilde{\psi}:[0, T]^{2} \times \mathcal{O} \rightarrow \mathcal{O}$ is defined as the solution of the initial value problem

$$
\left\{\begin{array}{l}
\frac{d}{d t} \widetilde{\boldsymbol{\psi}}(t ; s, \mathbf{x})=\mathbf{u}(\widetilde{\boldsymbol{\psi}}(t ; s, \mathbf{x}), t) \quad \forall t \in[0, T] \\
\widetilde{\boldsymbol{\psi}}(s ; s, \mathbf{x})=\mathbf{x}
\end{array}\right.
$$

It is well-known that the material derivative $D_{t} \mathbf{u}=\partial \mathbf{u} / \partial t+(\mathbf{u} \cdot \nabla) \mathbf{u}$ of $\mathbf{u}$ at instant $t_{0}$ satisfies:

$$
D_{t} \mathbf{u}\left(\mathbf{x}, t_{0}\right)=\frac{d}{d t}\left[\mathbf{u}\left(\widetilde{\boldsymbol{\psi}}\left(t ; t_{0}, \mathbf{x}\right), t\right)\right]_{\left.\right|_{t=t_{0}}} .
$$

Remark 2.2. By using a classical result of Liouville (see, for instance, [2, pp.251]), if

$$
\boldsymbol{\zeta} \in H^{2}(0, T)^{2}, \quad \omega \in H^{1}(0, T), \quad \mathbf{u} \in C([0, T] ; \widehat{\mathcal{K}}(\boldsymbol{\zeta}(t))),
$$

then we have that

$$
\operatorname{det} \mathbf{J}_{\widetilde{\psi}}=1
$$

where we have denoted by

$$
\mathbf{J}_{\widetilde{\psi}}=\left(\frac{\partial \widetilde{\psi}_{i}}{\partial y_{j}}\right)_{i, j}
$$

the jacobian matrix of the transformation $\mathbf{y} \mapsto \widetilde{\boldsymbol{\psi}}(\mathbf{y})$.

In the following lemma we give a weak formulation of the system (1.1)- 1.8 which will be then used to discretize the problem with respect to time.

Lemma 2.3. Assume that

$$
\begin{gathered}
\mathbf{u} \in L^{2}\left(0, T ; H^{2}(\Omega(t))^{2}\right) \cap H^{1}\left(0, T ; L^{2}(\Omega(t))^{2}\right) \cap C\left([0, T] ; H^{1}(\Omega(t))^{2}\right), \\
p \in L^{2}\left(0, T ; H^{1}(\Omega(t))\right), \quad \zeta \in H^{2}(0, T)^{2}, \quad \omega \in H^{1}(0, T)
\end{gathered}
$$

and that $\mathbf{u}$ is extended by

$$
\mathbf{u}(\mathbf{x}, t)=\boldsymbol{\zeta}^{\prime}(t)+\omega(t)(\mathbf{x}-\boldsymbol{\zeta}(t))^{\perp} \quad \forall \mathbf{x} \in B(\boldsymbol{\zeta}(t)) .
$$

Then $(\mathbf{u}, p, \boldsymbol{\zeta}, \omega)$ is the solution of 11.1$)-1.8$ if and only if for all $t \in[0, T], \mathbf{u}(\cdot, t) \in \mathcal{K}(\boldsymbol{\zeta}(t))$ $p(\cdot, t) \in M(\boldsymbol{\zeta}(t))$ and $(\mathbf{u}, p)$ satisfies

$$
\begin{gathered}
\left(\rho \frac{d}{d t}[\mathbf{u} \circ \widetilde{\boldsymbol{\psi}}](t), \boldsymbol{\varphi}\right)+a(\mathbf{u}, \boldsymbol{\varphi})+b(\boldsymbol{\varphi}, p)=(\rho \mathbf{f}(t), \boldsymbol{\varphi}) \quad \forall \boldsymbol{\varphi} \in \mathcal{K}(\boldsymbol{\zeta}(t)), \\
b(\mathbf{u}, q)=0 \quad \forall q \in M(\boldsymbol{\zeta}(t)),
\end{gathered}
$$

where

$$
a(\mathbf{u}, \mathbf{v})=2 \mu \int_{\mathcal{O}} \mathrm{D}(\mathbf{u}): \mathrm{D}(\mathbf{v}) \mathrm{d} \mathbf{x} \quad \forall \mathbf{u}, \mathbf{v} \in H^{1}(\mathcal{O})^{2}
$$

and

$$
b(\mathbf{u}, p)=-\int_{\mathcal{O}} \operatorname{div}(\mathbf{u}) p \mathrm{~d} \mathbf{x} \quad \forall \mathbf{u} \in H^{1}(\mathcal{O})^{2}, \forall p \in L_{0}^{2}(\mathcal{O}) .
$$

We skip the proof of Lemma 2.3 since it is similar to the proof of the corresponding result for the classical Navier-Stokes system (see, for instance, [16, Ch.12]). 


\section{Semi-discretization scheme and statement of the first main result}

By using the weak formulation (2.10) $-(2.11)$ we can derive a semi-discrete version of our system. For $N \in \mathbb{N}^{*}$ we denote $\Delta t=T / N$ and $t_{k}=k \Delta t$ for $k=0, \cdots, N$. Denote by $\left(\mathbf{u}^{k}, \boldsymbol{\zeta}^{k}\right) \in \widehat{\mathcal{K}}\left(\boldsymbol{\zeta}^{k}\right) \cap C^{0}(\overline{\mathcal{O}})^{2} \times \mathcal{O}$ the approximation of the solution of $(1.1)-(1.8)$ at the time $t=t_{k}$. In the sequel, we shall use the notation

$$
\widetilde{\mathbf{X}}(\mathbf{x})=\widetilde{\psi}\left(t_{k} ; t_{k+1}, \mathbf{x}\right) \quad \forall \mathbf{x} \in \mathcal{O} .
$$

We approximate the position of the rigid ball at instant $t_{k+1}$ by $\zeta^{k+1}$ which is defined by the relation

$$
\boldsymbol{\zeta}^{k+1}=\boldsymbol{\zeta}^{k}+\mathbf{u}^{k}\left(\boldsymbol{\zeta}^{k}\right) \Delta t
$$

We then define the characteristic function $\bar{\psi}$ associated with the semi-discretized velocity field as the solution of

$$
\left\{\begin{array}{l}
\frac{d}{d t} \overline{\boldsymbol{\psi}}\left(t ; t_{k+1}, \mathbf{x}\right)=\mathbf{u}^{k}\left(\overline{\boldsymbol{\psi}}\left(t ; t_{k+1}, \mathbf{x}\right)\right)-\mathbf{u}^{k}\left(\boldsymbol{\zeta}^{k}\right) \quad \forall t \in\left[t_{k}, t_{k+1}\right] \\
\overline{\boldsymbol{\psi}}\left(t_{k+1} ; t_{k+1}, \mathbf{x}\right)=\mathbf{x}-\mathbf{u}^{k}\left(\boldsymbol{\zeta}^{k}\right) \Delta t
\end{array}\right.
$$

and we denote

$$
\overline{\mathbf{X}}^{k}(\mathbf{x})=\overline{\boldsymbol{\psi}}\left(t_{k} ; t_{k+1}, \mathbf{x}\right) \quad \forall \mathbf{x} \in \mathcal{O} .
$$

In equation (3.3), the velocity $\mathbf{u}^{k}$ is extended by zero outside of the domain $\mathcal{O}$ as it was noted in Remark 2.1. This extension is necessary because we have $\overline{\mathbf{X}}^{k}(\mathcal{O}) \nsubseteq \mathcal{O}$. Indeed, we observe that due to the initial condition in $(3.3)$, if we consider $\mathbf{x} \in \mathcal{O}$, then $\overline{\boldsymbol{\psi}}\left(t_{k+1} ; t_{k+1}, \mathbf{x}\right)$ does not necessarily belong to $\mathcal{O}$. Nevertheless, one can easily check that $\overline{\mathbf{X}}^{k}(\mathcal{O}) \subseteq \mathcal{O}+$ $B\left(0,\left|\mathbf{u}^{k}\left(\boldsymbol{\zeta}^{k}\right)\right| \Delta t\right)$. We emphasize that the Cauchy problem $(3.3)$ is well-posed and then the characteristic function $\overline{\mathbf{X}}^{k}$ is also well defined. Indeed, since $\mathbf{u}^{k} \in H_{0}^{1}(\mathcal{O})^{2}$ with $\operatorname{div} \mathbf{u}^{k}=0$ in $\mathcal{O}$ and $\mathbf{u}^{k}=0$ in $\mathbb{R}^{2} \backslash \mathcal{O}$, the problem (3.3) admits a unique solution $\overline{\boldsymbol{\psi}}\left(\cdot ; t_{k+1}, \mathbf{x}\right) \in$ $C^{1}\left(\left[t_{k}, t_{k+1}\right]\right)$ for almost every $\mathbf{x} \in \mathbb{R}^{2}$, which satisfies the following measure preserving property (see [3, Section III]),

$$
\int_{\mathcal{A}} f\left(\overline{\boldsymbol{\psi}}\left(t ; t_{k+1}, \mathbf{x}\right)\right) \mathrm{d} \mathbf{x}=\int_{\overline{\boldsymbol{\psi}}\left(t ; t_{k+1}, \mathcal{A}\right)} f(\mathbf{y}) \mathrm{d} \mathbf{y}
$$

for all function $f \in L^{1}\left(\mathbb{R}^{2}\right)$ and for all $t \in\left[t_{k}, t_{k+1}\right]$. Moreover, since $\mathbf{u}^{k} \in C^{0}(\overline{\mathcal{O}})^{2}$, the caracteristic function $\overline{\boldsymbol{\psi}}\left(\cdot ; t_{k+1}, \mathbf{x}\right)$ is actually well defined in $\left[t_{k}, t_{k+1}\right]$, for all $\mathbf{x} \in \mathbb{R}^{2}$.

We next define $\mathbf{u}^{k+1} \in \widehat{\mathcal{K}}\left(\boldsymbol{\zeta}^{k+1}\right)$ as the solution of the following Stokes type system

$$
\left(\rho^{k+1} \frac{\mathbf{u}^{k+1}-\mathbf{u}^{k} \circ \overline{\mathbf{X}}^{k}}{\Delta t}, \boldsymbol{\varphi}\right)+a\left(\mathbf{u}^{k+1}, \boldsymbol{\varphi}\right)=\left(\rho^{k+1} \mathbf{f}^{k+1}, \boldsymbol{\varphi}\right) \quad \forall \boldsymbol{\varphi} \in \widehat{\mathcal{K}}\left(\boldsymbol{\zeta}^{k+1}\right),
$$

where $\mathbf{f}^{k+1}=\mathbf{f}\left(t_{k+1}\right)$ and $\rho^{k+1}$ is defined by

$$
\rho^{k+1}(\mathbf{x})= \begin{cases}\rho_{s} & \text { if } \mathbf{x} \in B\left(\boldsymbol{\zeta}^{k+1}\right), \\ \rho_{f} & \text { if } \mathbf{x} \in \mathcal{O} \backslash B\left(\boldsymbol{\zeta}^{k+1}\right) .\end{cases}
$$

The above equation can be rewritten by using a mixed formulation. It is clear that 3.6 is equivalent to the following system

$$
\begin{aligned}
\left(\rho^{k+1} \frac{\mathbf{u}^{k+1}-\mathbf{u}^{k} \circ \overline{\mathbf{X}}^{k}}{\Delta t}, \boldsymbol{\varphi}\right)+a\left(\mathbf{u}^{k+1}, \boldsymbol{\varphi}\right)+b\left(\boldsymbol{\varphi}, p^{k+1}\right) & \\
& =\left(\rho^{k+1} \mathbf{f}^{k+1}, \boldsymbol{\varphi}\right) \quad \forall \boldsymbol{\varphi} \in \mathcal{K}\left(\boldsymbol{\zeta}^{k+1}\right),
\end{aligned}
$$




$$
b\left(\mathbf{u}^{k+1}, q\right)=0 \quad \forall q \in M\left(\boldsymbol{\zeta}^{k+1}\right),
$$

of unknowns $\left(\mathbf{u}^{k+1}, p^{k+1}\right) \in \mathcal{K}\left(\boldsymbol{\zeta}^{k+1}\right) \times M\left(\boldsymbol{\zeta}^{k+1}\right)$.

It is well-known (see, for example, [6, Corollary I.4.1., pp.61]) that the mixed formulation (3.7)- 3.8 is a well-posed problem, provided that the spaces $\mathcal{K}(\boldsymbol{\zeta}), M(\boldsymbol{\zeta})$ and the bilinear form $b$ satisfy an inf-sup condition. The fact that this inf-sup condition is satisfied in our case follows from the result below.

Lemma 3.1. Suppose that $\boldsymbol{\zeta} \in \mathcal{O}$ is such that $d(\boldsymbol{\zeta}, \partial \mathcal{O})=1+\eta$, with $\eta>0$. Then there exists a constant $\beta>0$, depending only on $\eta$ and on $\mathcal{O}$, such that for all $q \in M(\zeta)$ there exists $\mathbf{u} \in \mathcal{K}(\boldsymbol{\zeta})$ with

$$
\int_{\mathcal{O}} \operatorname{div}(\mathbf{u}) q \mathrm{~d} \mathbf{x} \geq \beta\|\mathbf{u}\|_{H^{1}(\mathcal{O})^{2}}\|q\|_{L^{2}(\mathcal{O})} .
$$

The proof of the result above can be obtained by slightly modifying the approach used for the mixed formulation of the standard Stokes system (see, for instance [6, pp.81]), therefore it is left to the reader. In addition, it can be easily proved that $\mathbf{u}^{k+1}$ is continuous in $\overline{\mathcal{O}}$. To see this, we remark that $\left(\mathbf{u}^{k+1}, p^{k+1}\right)$ satisfies a Stokes problem in the fluid part $\mathcal{O} \backslash B\left(\boldsymbol{\zeta}^{k+1}\right)$ with a rigid velocity boundary condition on $\partial B\left(\boldsymbol{\zeta}^{k+1}\right)$. Then assuming $\mathbf{f}^{k+1} \in L^{2}(\mathcal{O})^{2}$, we get $\mathbf{u}^{k+1} \in H^{2}\left(\mathcal{O} \backslash B\left(\boldsymbol{\zeta}^{k+1}\right)\right)^{2}$ and we deduce that

$$
\mathbf{u}^{k+1} \in C^{0}(\overline{\mathcal{O}})^{2} .
$$

In the reminder of the paper, we suppose that $\mathbf{f}$ and $\mathbf{u}_{0}$ satisfy

$$
\begin{aligned}
& \mathbf{f} \in C\left([0, T] ; H^{1}(\mathcal{O})^{2}\right), \quad \mathbf{u}_{0} \in H^{2}(\Omega)^{2}, \quad \operatorname{div}\left(\mathbf{u}_{0}\right)=0 \quad \text { in } \Omega, \\
& \mathbf{u}_{0}=0 \quad \text { on } \partial \mathcal{O}, \quad \mathbf{u}_{0}(\mathbf{y})=\boldsymbol{\zeta}_{1}+\omega_{0}\left(\mathbf{y}-\boldsymbol{\zeta}_{0}\right)^{\perp} \quad \text { on } \partial B\left(\boldsymbol{\zeta}_{0}\right),
\end{aligned}
$$

where $\boldsymbol{\zeta}_{0}, \boldsymbol{\zeta}_{1} \in \mathbb{R}^{2}, \omega_{0} \in \mathbb{R}$ and $\Omega=\mathcal{O} \backslash B\left(\boldsymbol{\zeta}_{0}\right)$. Let us also assume that the corresponding solution $(\mathbf{u}, p, \boldsymbol{\zeta}, \omega)$ of problem (1.1)- 1.8$)$ satisfies

$$
\left\{\begin{array}{l}
\mathbf{u} \in C\left([0, T] ; H^{2}(\Omega(t))^{2}\right) \cap H^{1}\left(0, T ; L^{2}(\Omega(t))^{2}\right), \\
D_{t}^{2} \mathbf{u} \in L^{2}\left(0, T ; L^{2}(\Omega(t))^{2}\right), \quad \mathbf{u} \in C\left([0, T] ; C^{0,1}(\overline{\mathcal{O}})^{2}\right) \\
p \in C\left([0, T] ; H^{1}(\Omega(t))\right), \quad \zeta \in H^{3}(0, T)^{2}, \quad \omega \in H^{2}(0, T)
\end{array}\right.
$$

and

$$
\operatorname{dist}(B(\boldsymbol{\zeta}(t)), \partial \mathcal{O})>0 \quad \forall t \in[0, T] .
$$

Remark 3.2. The hypotheses $(3.12)$ and $(3.13)$ imply the existence of $\eta>0$ such that

$$
\operatorname{dist}(B(\boldsymbol{\zeta}(t)), \partial \mathcal{O})>3 \eta \quad \forall t \in[0, T] .
$$

Let us now state our first main result concerning the convergence of the semi-discrete scheme $(3.7)-(3.8)$ :

Theorem 3.3. Suppose that $\mathcal{O}$ is an open smooth bounded domain in $\mathbb{R}^{2}, \mathbf{f}$ and $\mathbf{u}_{0}$ satisfy (3.11) and $(\mathbf{u}, p, \boldsymbol{\zeta}, \omega)$ is a solution of (1.1)-(1.8) satisfying (3.12) $-(3.13)$. Then there exist two positive constants $C$ and $\tau^{*}$ not depending on $\Delta t$ such that for all $0<\Delta t \leqslant \tau^{*}$ the solution $\left(\mathbf{u}^{k}, p^{k}, \boldsymbol{\zeta}^{k}\right)$ of the semi-discretization problem (3.7)-(3.8) satisfies

$$
\sup _{1 \leqslant k \leqslant N}\left(\left|\boldsymbol{\zeta}\left(t_{k}\right)-\boldsymbol{\zeta}^{k}\right|+\left\|\mathbf{u}\left(t_{k}\right)-\mathbf{u}^{k}\right\|_{L^{2}(\mathcal{O})^{2}}\right) \leqslant C \Delta t .
$$

\section{Fully discrete formulation and statement of the second main result}

In order to discretize the problem $(3.7)-(3.8)$ with respect to the space variable, we introduce two families of finite element spaces which approximate the spaces $\mathcal{K}(\boldsymbol{\zeta})$ and $M(\boldsymbol{\zeta})$ defined in (2.2) and (2.4). To this end, we consider the discretization parameter $0<h<1$. 
Let $\mathcal{T}_{h}$ be a quasi-uniform triangulation of the domain $\mathcal{O}$. We denote by $\mathcal{W}_{h}$ the $\mathbb{P}_{1^{-}}$ bubble finite elements space associated with $\mathcal{T}_{h}$ for the velocity field in the Stokes problem and by $E_{h}$ the $\mathbb{P}_{1}$-finite elements space for the pressure. Then, we define the following finite elements spaces for a conform approximation of the fluid-rigid system:

$$
\begin{aligned}
\mathcal{K}_{h}(\boldsymbol{\zeta}) & =\mathcal{W}_{h} \cap \mathcal{K}(\boldsymbol{\zeta}) & & \forall \boldsymbol{\zeta} \in \mathcal{O} \\
M_{h}(\boldsymbol{\zeta}) & =E_{h} \cap M(\boldsymbol{\zeta}) & & \forall \boldsymbol{\zeta} \in \mathcal{O} .
\end{aligned}
$$

In order to define the approximate characteristics, let us denote by $F_{h}$ the $\mathbb{P}_{2}$-finite element space associated with the triangulation $\mathcal{T}_{h}$ and we introduce the space:

$$
\mathcal{R}_{h}(\boldsymbol{\zeta})=\left\{\nabla^{\perp} \varphi_{h}: \varphi_{h} \in F_{h}, \varphi_{h}=0 \text { on } \partial \mathcal{O}\right\} \cap \mathcal{K}(\boldsymbol{\zeta}) \quad \forall \boldsymbol{\zeta} \in \mathcal{O},
$$

where we have denoted by $\nabla^{\perp} \varphi_{h}=\left(\begin{array}{c}-\frac{\partial \varphi_{h}}{\partial y} \\ \frac{\partial \varphi_{h}}{\partial x}\end{array}\right)$.

We denote $\mathbf{P}(\boldsymbol{\zeta})$ the orthogonal projection from $L^{2}(\mathcal{O})^{2}$ onto $\mathcal{R}_{h}(\boldsymbol{\zeta})$, i.e. if $\mathbf{u} \in L^{2}(\mathcal{O})^{2}$ then $\mathbf{P}(\boldsymbol{\zeta}) \mathbf{u} \in \mathcal{R}_{h}(\boldsymbol{\zeta})$ such that $\left(\mathbf{u}-\mathbf{P}(\boldsymbol{\zeta}) \mathbf{u}, \mathbf{r}_{h}\right)=0$ for all $\mathbf{r}_{h} \in \mathcal{R}_{h}(\boldsymbol{\zeta})$.

Let $N$ be a positive integer. We denote $\Delta t=T / N$ and $t_{k}=k \Delta t$ for all $k \in\{0, \ldots, N\}$. Assume that the approximate solution $\left(\mathbf{u}_{h}^{k}, p_{h}^{k}, \boldsymbol{\zeta}_{h}^{k}\right)$ of (1.1)-(1.8) at $t=t_{k}$ is known. We describe below the numerical scheme allowing to determinate the approximate solution $\left(\mathbf{u}_{h}^{k+1}, p_{h}^{k+1}, \boldsymbol{\zeta}_{h}^{k+1}\right)$ at $t=t_{k+1}$. First, we compute $\boldsymbol{\zeta}_{h}^{k+1} \in \mathbb{R}^{2}$ by

$$
\boldsymbol{\zeta}_{h}^{k+1}=\boldsymbol{\zeta}_{h}^{k}+\mathbf{u}_{h}^{k}\left(\boldsymbol{\zeta}_{h}^{k}\right) \Delta t .
$$

We consider the approximated characteristic function $\overline{\boldsymbol{\psi}}_{h}^{k}$ defined as the solution of

$$
\left\{\begin{array}{l}
\frac{d}{d t} \overline{\boldsymbol{\psi}}_{h}^{k}\left(t ; t_{k+1}, \mathbf{x}\right)=\mathbf{P}\left(\boldsymbol{\zeta}_{h}^{k}\right) \mathbf{u}_{h}^{k}\left(\overline{\boldsymbol{\psi}}_{h}^{k}\left(t ; t_{k+1}, \mathbf{x}\right)\right)-\mathbf{P}\left(\boldsymbol{\zeta}_{h}^{k}\right) \mathbf{u}_{h}^{k}\left(\boldsymbol{\zeta}_{h}^{k}\right) \quad \forall t \in\left[t_{k}, t_{k+1}\right] \\
\overline{\boldsymbol{\psi}}_{h}^{k}\left(t_{k+1} ; t_{k+1}, \mathbf{x}\right)=\mathbf{x}-\mathbf{u}_{h}^{k}\left(\boldsymbol{\zeta}_{h}^{k}\right) \Delta t
\end{array}\right.
$$

Finally, we define

$$
\overline{\mathbf{X}}_{h}^{k}(\mathbf{x})=\overline{\boldsymbol{\psi}}_{h}^{k}\left(t_{k} ; t_{k+1}, \mathbf{x}\right) \quad \forall \mathbf{x} \in \mathcal{O} .
$$

We remark that, since $\operatorname{div}\left(\mathbf{P}\left(\boldsymbol{\zeta}_{h}^{k}\right) \mathbf{u}_{h}^{k}\left(\overline{\boldsymbol{\psi}}_{h}^{k}\left(t ; t_{k+1}, \cdot\right)\right)-\mathbf{P}\left(\boldsymbol{\zeta}_{h}^{k}\right) \mathbf{u}_{h}^{k}\left(\boldsymbol{\zeta}_{h}^{k}\right)\right)=0$ and $\nabla\left(\mathbf{x}-\mathbf{u}_{h}^{k}\left(\boldsymbol{\zeta}_{h}^{k}\right) \Delta t\right)=\mathbf{I d}$, we get

$$
\operatorname{det} \mathbf{J}_{\bar{\psi}_{h}^{k}}=1 \text {. }
$$

In the sequel, we shall split the mesh into the union of 4 different types of triangle's subsets. We first introduce $A_{h}$ as the union of all triangles intersecting the ball $B\left(\boldsymbol{\zeta}_{h}^{k}\right)$, i.e.

$$
A_{h}=\bigcup_{\substack{T \in \mathcal{T}_{h} \\ T \cap B\left(\zeta_{h}^{k}\right) \neq \emptyset}} T .
$$

We also denote by $Q_{h}$ the union of all triangles such that all their vertices are contained in $\overline{A_{h}}$. The triangles of $\mathcal{T}_{h}$ are then splitted into the 4 following categories (see Figure 1 ):

- $\mathcal{F}_{1}$ is the subset of $\mathcal{T}_{h}$ formed by all triangles $T \in \mathcal{T}_{h}$ such that $\bar{T} \subset B\left(\boldsymbol{\zeta}_{h}^{k}\right)$.

- $\mathcal{F}_{2}$ is the subset formed by all triangles $T \in \mathcal{T}_{h} \backslash \mathcal{F}_{1}$ such that $\bar{T} \subset \overline{Q_{h}}$.

- $\mathcal{F}_{3}$ is the subset formed by all triangles $T \in \mathcal{T}_{h}$ such that $\bar{T} \cap \overline{Q_{h}} \neq \emptyset$ and $T \not \subset \overline{Q_{h}}$.

- $\mathcal{F}_{4}=\mathcal{T}_{h} \backslash\left(\mathcal{F}_{1} \cup \mathcal{F}_{2} \cup \mathcal{F}_{3}\right)$.

We introduce two approximated density functions $\rho_{h}^{k}$ and $\bar{\rho}_{h}^{k}$ as follows:

$$
\rho_{h}^{k}(\mathbf{x})= \begin{cases}\rho_{s} & \text { if } \mathbf{x} \in B\left(\boldsymbol{\zeta}_{h}^{k}\right), \\ \rho_{f} & \text { if } \mathbf{x} \in \mathcal{O} \backslash B\left(\boldsymbol{\zeta}_{h}^{k}\right)\end{cases}
$$


This triangle does not belong to $A_{h}$ but lies in $Q_{h}$ since its three vertices are in $\overline{A_{h}}$.

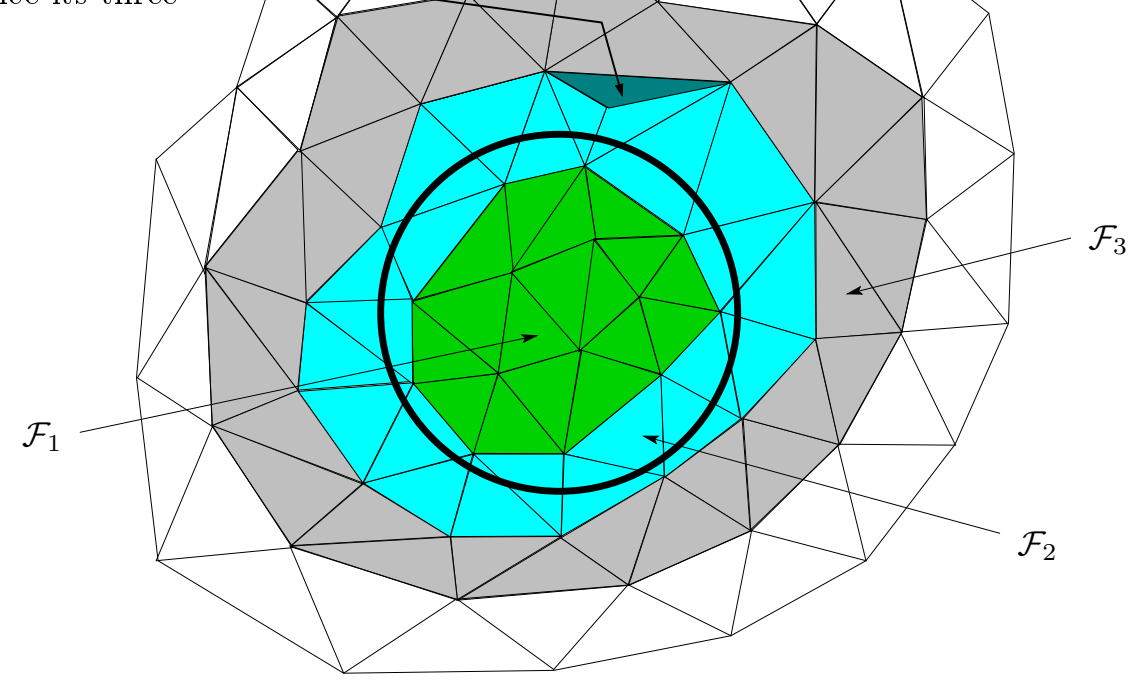

Figure 1: The rigid ball and the related splitting of the triangulation.

and $\bar{\rho}_{h}^{k}$ is the continuous function in $\overline{\mathcal{O}}$ which is piecewise linear on triangles of $\mathcal{T}_{h}$ and satisfies

$$
\bar{\rho}_{h}^{k}(\mathbf{x})= \begin{cases}\rho_{s} & \text { if } \mathbf{x} \in \overline{Q_{h}}, \\ \rho_{f} & \text { if } \mathbf{x} \in \mathcal{F}_{4} .\end{cases}
$$

With these notations, we consider the following mixed variational fully discrete formulation: Find $\left(\mathbf{u}_{h}^{k+1}, p_{h}^{k+1}\right) \in \mathcal{K}_{h}\left(\boldsymbol{\zeta}_{h}^{k+1}\right) \times M_{h}\left(\boldsymbol{\zeta}_{h}^{k+1}\right)$ such that

$$
\begin{gathered}
\left(\rho_{h}^{k+1} \frac{\mathbf{u}_{h}^{k+1}-\mathbf{u}_{h}^{k} \circ \overline{\mathbf{X}}_{h}^{k}}{\Delta t}, \boldsymbol{\varphi}\right)+a\left(\mathbf{u}_{h}^{k+1}, \boldsymbol{\varphi}\right)+b\left(\boldsymbol{\varphi}, p_{h}^{k+1}\right) \\
=\left(\bar{\rho}_{h}^{k+1} \mathbf{f}_{h}^{k+1}, \boldsymbol{\varphi}\right) \quad \forall \boldsymbol{\varphi} \in \mathcal{K}_{h}\left(\boldsymbol{\zeta}_{h}^{k+1}\right), \\
b\left(\mathbf{u}_{h}^{k+1}, q\right)=0 \quad \forall q \in M_{h}\left(\boldsymbol{\zeta}_{h}^{k+1}\right),
\end{gathered}
$$

where $\mathbf{f}_{h}^{k+1}$ is the $L^{2}(\mathcal{O})^{2}$-projection of $\mathbf{f}^{k+1}=\mathbf{f}\left(t_{k+1}\right)$ on $\left(E_{h}\right)^{2}$.

Let us now state the second main result of this paper which asserts the convergence of the fully-discrete scheme (4.7)- 4.8):

Theorem 4.1. Let $\mathcal{O}$ be a convex domain with a polygonal boundary. Suppose that $\mathbf{f}$ and $\mathbf{u}_{0}$ satisfy the conditions (3.11) and that $(\mathbf{u}, p, \boldsymbol{\zeta}, \omega)$ is a solution of (1.1)-1.8) satisfying the regularity properties (3.12) and such that (3.13) holds. Let $C_{0}>0$ and $0<\alpha \leq 1$ be two fixed constants. Then there exist two positive constants $C$ and $\tau^{*}$ independent of $h$ and $\Delta t$ such that for all $0<\Delta t \leq \tau^{*}$ and for all $h \leq C_{0} \Delta t^{1+\alpha}$ we have

$$
\sup _{1 \leq k \leq N}\left(\left|\boldsymbol{\zeta}\left(t_{k}\right)-\boldsymbol{\zeta}_{h}^{k}\right|+\left\|\mathbf{u}\left(t_{k}\right)-\mathbf{u}_{h}^{k}\right\|_{L^{2}(\mathcal{O})^{2}}\right) \leq C \Delta t^{\alpha} .
$$

Remark 4.2. In order to get an approximation of first order in time (i.e. $O(\Delta t)$ ), we have to choose $\alpha=1$. In this case, the corresponding condition on $h$ becomes $h \leq C_{0} \Delta t^{2}$ which is similar to the one obtained in [18, Th.3.2] in the case of equal densities $\rho_{f}=\rho_{s}$.

\section{Properties on the characteristic function}

In this section, we prove some properties on the new characteristic function which are essential for the proof of our main results. 
Lemma 5.1. For any free divergence velocity field $\mathbf{v} \in H_{0}^{1}(\mathcal{O})^{2} \cap C^{0}(\overline{\mathcal{O}})^{2}$ extended by zero outside of $\mathcal{O}$, and for any differentiable function $\mathbf{R}: \mathcal{O} \rightarrow \mathbb{R}^{2}$ such that $\operatorname{det}(\nabla \mathbf{R})=1$ and $\mathbf{R}\left(\bar{S}_{k+1}\right)=\bar{S}_{k}$, where $S_{k}$ and $S_{k+1}$ are two open smooth subsets of $\mathcal{O}$, we consider the characteristic function as the solution of problem

$$
\left\{\begin{array}{l}
\frac{d}{d t} \boldsymbol{\psi}\left(t ; t_{k+1}, \mathbf{x}\right)=\mathbf{v}\left(\boldsymbol{\psi}\left(t ; t_{k+1}, \mathbf{x}\right)\right) \quad \forall t \in\left[t_{k}, t_{k+1}\right] \\
\boldsymbol{\psi}\left(t_{k+1} ; t_{k+1}, \mathbf{x}\right)=\mathbf{R}(\mathbf{x})
\end{array}\right.
$$

and we denote

$$
\mathbf{X}(\mathbf{x})=\boldsymbol{\psi}\left(t_{k} ; t_{k+1}, \mathbf{x}\right) \quad \forall \mathbf{x} \in \mathcal{O} .
$$

If $\mathbf{v}(\mathbf{z}) \cdot \mathbf{n}=0$ for any $\mathbf{z} \in \partial S_{k}$, then the characteristic function satisfies the following properties:

i) $\mathbf{X}\left(\bar{S}_{k+1}\right)=\bar{S}_{k}$;

ii) For any $f \in L^{2}\left(\mathbb{R}^{2}\right)$ such that $f=0$ in $\mathbb{R}^{2} \backslash \mathcal{O}$, we have

$$
\left\|f \circ \boldsymbol{\psi}\left(t ; t_{k+1}, \cdot\right)\right\|_{L^{2}(\mathcal{O})} \leq\|f\|_{L^{2}(\mathcal{O})} \quad \forall t \in\left[t_{k}, t_{k+1}\right] .
$$

Proof. Let us first remark that the Cauchy problem (5.1) is well-posed. To see this, we transform problem (5.1) by making use of the following change of unknown:

$$
\psi\left(t ; t_{k+1}, \mathbf{x}\right)=\varphi\left(t ; t_{k+1}, \mathbf{R}(\mathbf{x})\right)
$$

where $\varphi$ satisfies

$$
\left\{\begin{array}{l}
\frac{d}{d t} \boldsymbol{\varphi}\left(t ; t_{k+1}, \mathbf{y}\right)=\mathbf{v}\left(\boldsymbol{\varphi}\left(t ; t_{k+1}, \mathbf{y}\right)\right) \quad \forall t \in\left[t_{k}, t_{k+1}\right], \\
\boldsymbol{\varphi}\left(t_{k+1} ; t_{k+1}, \mathbf{y}\right)=\mathbf{y} \quad \forall \mathbf{y} \in \mathbb{R}^{2} .
\end{array}\right.
$$

According to [3, Section III], the Cauchy problem (5.5) admits a unique solution $\varphi\left(\cdot ; t_{k+1}, \mathbf{y}\right) \in$ $C^{1}(\mathbb{R})^{2}$ for almost every $\mathbf{y} \in \mathbb{R}^{2}$ and satisfies the following measure preserving property

$$
\int_{\mathcal{A}} F\left(\varphi\left(t ; t_{k+1}, \mathbf{y}\right)\right) \mathrm{d} \mathbf{y}=\int_{\varphi\left(t ; t_{k+1}, \mathcal{A}\right)} F(\mathbf{x}) \mathrm{d} \mathbf{x},
$$

for any subset $\mathcal{A} \subset \mathbb{R}^{2}$, for all function $F \in L^{1}\left(\mathbb{R}^{2}\right)$ and for all $t \in\left[t_{k}, t_{k+1}\right]$. Since the velocity field $\mathbf{v}$ is continuous in $\mathbb{R}^{2}$, then $\varphi\left(\cdot ; t_{k+1}, \mathbf{y}\right)$ is actually defined for all $\mathbf{y} \in \mathbb{R}^{2}$. Moreover, due to the hypothesis $\mathbf{v} \cdot \mathbf{n}=0$ on $\partial S_{k}$, we have that $\varphi\left(t_{k} ; t_{k+1}, \cdot\right)$ maps $\bar{S}_{k}$ onto itself (see [3, Section IV]).

We can now prove the equality $i$ ). In fact, we have that

$$
\mathbf{X}\left(\bar{S}_{k+1}\right)=\boldsymbol{\varphi}\left(t_{k} ; t_{k+1}, \mathbf{R}\left(\bar{S}_{k+1}\right)\right)=\boldsymbol{\varphi}\left(t_{k} ; t_{k+1}, \bar{S}_{k}\right)=\bar{S}_{k} .
$$

Let us turn to the proof of $i$ ). Under the assumption $\operatorname{det}(\nabla \mathbf{R})=1$ and using the property (5.6), we obtain

$$
\begin{aligned}
\left\|f \circ \boldsymbol{\psi}\left(t ; t_{k+1}, \cdot\right)\right\|_{L^{2}(\mathcal{O})}^{2} & =\int_{\mathcal{O}}\left|f\left(\boldsymbol{\psi}\left(t ; t_{k+1}, \mathbf{x}\right)\right)\right|^{2} \mathrm{~d} \mathbf{x}=\int_{\mathcal{O}}\left|f\left(\varphi\left(t ; t_{k+1}, \mathbf{R}(\mathbf{x})\right)\right)\right|^{2} \mathrm{~d} \mathbf{x} \\
& =\int_{\mathbf{R}(\mathcal{O})}\left|f\left(\varphi\left(t ; t_{k+1}, \mathbf{y}\right)\right)\right|^{2} \mathrm{~d} \mathbf{y}=\int_{\boldsymbol{\psi}\left(t ; t_{k+1}, \mathcal{O}\right)}|f(\mathbf{z})|^{2} \mathrm{~d} \mathbf{z} .
\end{aligned}
$$

On the other hand, since $f=0$ in $\mathbb{R}^{2} \backslash \mathcal{O}$ we have

$$
\int_{\boldsymbol{\psi}\left(t ; t_{k+1}, \mathcal{O}\right)}|f(\mathbf{z})|^{2} \mathrm{~d} \mathbf{z}=\int_{\boldsymbol{\psi}\left(t ; t_{k+1}, \mathcal{O}\right) \cap \mathcal{O}}|f(\mathbf{z})|^{2} \mathrm{~d} \mathbf{z} \leq \int_{\mathcal{O}}|f(\mathbf{z})|^{2} \mathrm{~d} \mathbf{z} .
$$

Therefore, we conclude the result ii). 
In the sequel, we state two corollaries of the above lemma which state the properties on the characteristic functions associated with the semi-discretized and fully-discretized velocity fields:

Corollary 5.2. For any $k \in\{0, \ldots, N\}$, the characteristic function $\bar{\psi}$ defined in $(3.3)-(3.4)$ satisfies the following properties:

i) $\overline{\mathbf{X}}^{k}\left(B\left(\boldsymbol{\zeta}^{k+1}\right)\right)=B\left(\boldsymbol{\zeta}^{k}\right)$;

ii) If we extend by $\rho_{f}$ the density field $\rho^{k}$ outside of $\mathcal{O}$, we have

$$
\rho^{k+1}=\rho^{k} \circ \overline{\mathbf{X}}^{k}
$$

iii) For any $f \in L^{2}\left(\mathbb{R}^{2}\right)$ such that $f=0$ in $\mathbb{R}^{2} \backslash \mathcal{O}$, we have

$$
\left\|f \circ \overline{\boldsymbol{\psi}}\left(t ; t_{k+1}, \cdot\right)\right\|_{L^{2}(\mathcal{O})} \leq\|f\|_{L^{2}(\mathcal{O})} \quad \forall t \in\left[t_{k}, t_{k+1}\right] .
$$

Proof. The properties $i)$ and iii) are direct consequences of Lemma 5.1. In fact, we have that the function $\mathbf{R}(\mathbf{x})=\mathbf{x}-\mathbf{u}^{k}\left(\boldsymbol{\zeta}^{k}\right) \Delta t$ maps $B\left(\boldsymbol{\zeta}^{k+1}\right)$ onto $B\left(\boldsymbol{\zeta}^{k}\right), \nabla \mathbf{R}=\mathbf{I d}$. Moreover, the velocity field

$$
\mathbf{v}(\mathbf{z})=\mathbf{u}^{k}(\mathbf{z})-\mathbf{u}^{k}\left(\boldsymbol{\zeta}^{k}\right) \quad \forall \mathbf{z} \in \mathbb{R}^{2}
$$

has free divergence and for any $\mathbf{z} \in B\left(\boldsymbol{\zeta}^{k}\right)$ the decomposition 2.5 allows us to get that

$$
\mathbf{v}(\mathbf{z})=\omega_{\mathbf{u}^{k}}\left(\mathbf{z}-\boldsymbol{\zeta}^{k}\right)^{\perp},
$$

which implies that the hypothesis $\mathbf{v} \cdot \mathbf{n}=0$ on $\partial B\left(\boldsymbol{\zeta}^{k}\right)$ holds.

The equality ii) is a direct consequence of $i$ ) and the extension of $\rho^{k}$ by $\rho_{f}$ outside of $\mathcal{O}$. In fact, we have $\left(\rho^{k} \circ \overline{\mathbf{X}}^{k}\right)(\mathbf{x})=\rho_{s}$ if and only if $\overline{\mathbf{X}}^{k}(\mathbf{x}) \in B\left(\boldsymbol{\zeta}^{k}\right)$ which is equivalent to $\mathbf{x} \in B\left(\zeta^{k+1}\right)$ due to identity $\left.i\right)$.

Corollary 5.3. For any $k \in\{0, \ldots, N\}$ and $h \in(0,1)$, the characteristic function $\overline{\boldsymbol{\psi}}_{h}^{k}$ defined in 4.2) -4.3) satisfies the following properties:

i) $\overline{\mathbf{X}}_{h}^{k}\left(B\left(\boldsymbol{\zeta}_{h}^{k+1}\right)\right)=B\left(\boldsymbol{\zeta}_{h}^{k}\right)$;

ii) If we extend by $\rho_{f}$ the density field $\rho_{h}^{k}$ outside of $\mathcal{O}$, we have

$$
\rho_{h}^{k+1}=\rho_{h}^{k} \circ \overline{\mathbf{X}}_{h}^{k}
$$

iii) For any $f \in L^{2}\left(\mathbb{R}^{2}\right)$ such that $f=0$ in $\mathbb{R}^{2} \backslash \mathcal{O}$, we have

$$
\left\|f \circ \overline{\boldsymbol{\psi}}_{h}^{k}\left(t ; t_{k+1}, \cdot\right)\right\|_{L^{2}(\mathcal{O})^{2}} \leq\|f\|_{L^{2}(\mathcal{O})^{2}} \quad \forall t \in\left[t_{k}, t_{k+1}\right] .
$$

Proof. The proof is similar to the proof of Corollary 5.2. It is enough to observe that the initial condition from equation 4.2$), \mathbf{R}(\mathbf{x})=\mathbf{x}-\mathbf{u}_{h}^{k}\left(\boldsymbol{\zeta}_{h}^{k}\right) \Delta t$ maps $B\left(\boldsymbol{\zeta}_{h}^{k+1}\right)$ onto $B\left(\boldsymbol{\zeta}_{h}^{k}\right)$ and $\nabla \mathbf{R}=\mathbf{I d}$. The velocity field

$$
\mathbf{v}(\mathbf{z})=\mathbf{P}\left(\boldsymbol{\zeta}_{h}^{k}\right) \mathbf{u}_{h}^{k}(\mathbf{z})-\mathbf{P}\left(\boldsymbol{\zeta}_{h}^{k}\right) \mathbf{u}_{h}^{k}\left(\boldsymbol{\zeta}_{h}^{k}\right) \quad \forall \mathbf{z} \in \mathbb{R}^{2}
$$

has free divergence and for any $\mathbf{z} \in B\left(\boldsymbol{\zeta}_{h}^{k}\right)$ the decomposition 2.5 gives us

$$
\mathbf{v}(\mathbf{z})=\omega_{\mathbf{P}\left(\boldsymbol{\zeta}_{h}^{k}\right) \mathbf{u}_{h}^{k}}\left(\mathbf{z}-\boldsymbol{\zeta}_{h}^{k}\right)^{\perp},
$$

where $\omega_{\mathbf{P}\left(\boldsymbol{\zeta}_{h}^{k}\right) \mathbf{u}_{h}^{k}}$ is the angular velocity associated with the rigid velocity field $\mathbf{P}\left(\boldsymbol{\zeta}_{h}^{k}\right) \mathbf{u}_{h}^{k}$ in $B\left(\boldsymbol{\zeta}_{h}^{k}\right)$. This implies that $\mathbf{v} \cdot \mathbf{n}=0$ on $\partial B\left(\boldsymbol{\zeta}_{h}^{k}\right)$. With these remarks, the hypotheses of Lemma 5.1 are satisfied and thus the proof is concluded. 
Remark 5.4. In the case of a general rigid body, not necessarily ball, the definition of the characteristic function $\bar{\psi}$ has to be modified in order to take into account the rotation effects. To this end, we denote by $B\left(\boldsymbol{\zeta}^{k}, \theta^{k}\right)$ the rigid body with the center of mass $\boldsymbol{\zeta}^{k}$ and the orientation angle $\theta^{k}$. We also denote $\omega_{k}$ the approximate angular velocity and $\mathcal{R}_{\theta}$ will stand for the rotation matrix of angle $\theta$. The characteristic function $\bar{\psi}$ is now defined as the solution of

$$
\left\{\begin{array}{l}
\frac{d}{d t} \overline{\boldsymbol{\psi}}\left(t ; t_{k+1}, \mathbf{x}\right)=\mathbf{u}^{k}\left(\overline{\boldsymbol{\psi}}\left(t ; t_{k+1}, \mathbf{x}\right)\right)-\mathbf{u}_{R}^{k}\left(\overline{\boldsymbol{\psi}}\left(t ; t_{k+1}, \mathbf{x}\right)\right), \\
\overline{\boldsymbol{\psi}}\left(t_{k+1} ; t_{k+1}, \mathbf{x}\right)=\mathcal{R}_{-\omega_{k}\left(t_{k+1}-t_{k}\right)}\left(\mathbf{x}-\boldsymbol{\zeta}^{k+1}\right)+\boldsymbol{\zeta}^{k}
\end{array}\right.
$$

where $\mathbf{u}^{k}$ is extended by zero outside of $\mathcal{O}$ as in Remark 2.1 and $\mathbf{u}_{R}^{k}$ is the rigid velocity field defined as follows

$$
\mathbf{u}_{R}^{k}(\mathbf{x})=\mathbf{u}^{k}\left(\boldsymbol{\zeta}^{k}\right)+\omega_{k}\left(\mathbf{x}-\boldsymbol{\zeta}^{k}\right)^{\perp} \quad \forall \mathbf{x} \in \mathbb{R}^{2} .
$$

We also define the function $\overline{\mathbf{X}}^{k}$ by

$$
\overline{\mathbf{X}}^{k}(\mathbf{x})=\overline{\boldsymbol{\psi}}\left(t_{k} ; t_{k+1}, \mathbf{x}\right) \quad \forall \mathbf{x} \in \mathcal{O} .
$$

With these definitions, the hypotheses of Lemma 5.1 are still fulfilled and then Corollary 5.2 holds for the general case of a rigid body.

\section{Proof of the first main result}

Let us now prove our first main result stated in Theorem 3.3 concerning the convergence of the semi-discretization scheme (3.7)-(3.8). For this purpose, we first introduce the transformed system (6.4)-6.5) below, and then in Subsection 6.2 we give the proof of the convergence result.

\subsection{Transformed system}

We need to compare the exact solution $\mathbf{u}\left(t_{k}\right) \in \mathcal{K}\left(\boldsymbol{\zeta}\left(t_{k}\right)\right)$, which is a rigid velocity field in $B\left(\boldsymbol{\zeta}\left(t_{k}\right)\right)$ with $\mathbf{u}^{k} \in \mathcal{K}\left(\boldsymbol{\zeta}^{k}\right)$ which is a rigid velocity field in $B\left(\boldsymbol{\zeta}^{k}\right)$. To this end, we use the change of variable $X_{\zeta_{1}, \zeta_{2}}$ defined in [18, Section 5], which maps the ball $B\left(\boldsymbol{\zeta}_{1}\right)$ into the ball $B\left(\boldsymbol{\zeta}_{2}\right)$. We define

$$
\mathbf{X}^{k}=\mathbf{X}_{\boldsymbol{\zeta}^{k}, \boldsymbol{\zeta}\left(t_{k}\right)}, \quad \mathbf{Y}^{k}=\mathbf{Y}_{\boldsymbol{\zeta}^{k}, \boldsymbol{\zeta}\left(t_{k}\right)},
$$

where $Y_{\boldsymbol{\zeta}_{1}, \boldsymbol{\zeta}_{2}}$ is the inverse mapping of $X_{\boldsymbol{\zeta}_{1}, \boldsymbol{\zeta}_{2}}$. We also define

$$
\mathbf{U}^{k}(\mathbf{y})=\mathbf{J}_{\mathbf{Y}^{k}}\left(\mathbf{X}^{k}(\mathbf{y})\right) \mathbf{u}\left(\mathbf{X}^{k}(\mathbf{y}), t_{k}\right), \quad P^{k}(\mathbf{y})=p\left(\mathbf{X}^{k}(\mathbf{y}), t_{k}\right),
$$

where $\mathbf{J}_{\mathbf{Y}^{k}}$ is the determinant of the jacobian matrix of $\mathbf{Y}^{k}$. We recall that $\mathbf{U}^{k} \in \widehat{\mathcal{K}}\left(\boldsymbol{\zeta}^{k}\right)$ and $P^{k} \in M\left(\boldsymbol{\zeta}^{k}\right)$. Let us introduce the following notations that will be useful in the sequel:

$$
\widehat{\mathbf{X}}=\mathbf{Y}^{k} \circ \widetilde{\mathbf{X}} \circ \mathbf{X}^{k+1}
$$

and

$$
\widehat{\mathbf{J}}=\left(\mathbf{J}_{\mathbf{Y}^{k+1}} \circ \mathbf{X}^{k+1}\right)\left(\mathbf{J}_{\mathbf{X}^{k}} \circ \widehat{\mathbf{X}}\right),
$$

where $\widetilde{\mathbf{X}}$ is defined in 3.1 . The characteristics functions satisfy the properties depicted on the following diagram:

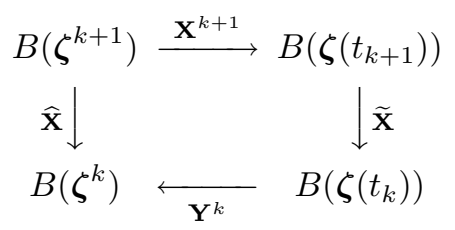

We point out that the following relation holds

$$
\rho^{k+1}=\rho^{k} \circ \widehat{\mathbf{X}} .
$$


The transformed functions $\mathbf{U}^{k+1}$ and $P^{k+1}$ satisfy a mixed weak formulation with test functions in $\mathcal{K}\left(\zeta^{k+1}\right)$ and $M\left(\zeta^{k+1}\right)$. Precisely, we have the following result which can be obtained as in [18, Proposition 6.2] with a very slight modification of the proof.

Proposition 6.1. The functions $\left(\mathbf{U}^{k+1}, P^{k+1}\right)$ defined by 6.1 satisfy

$$
\begin{aligned}
\frac{1}{\Delta t}\left(\rho^{k+1}\left[\mathbf{U}^{k+1}-\widehat{\mathbf{J}}\left(\mathbf{U}^{k} \circ \widehat{\mathbf{X}}\right)\right], \boldsymbol{\varphi}\right)+ & a\left(\mathbf{U}^{k+1}, \boldsymbol{\varphi}\right)+b\left(\boldsymbol{\varphi}, P^{k+1}\right) \\
& =\left(\rho^{k+1} \mathbf{f}^{k+1}, \boldsymbol{\varphi}\right)+\left(\mathbf{A}_{k}, \boldsymbol{\varphi}\right) \quad \forall \boldsymbol{\varphi} \in \mathcal{K}\left(\boldsymbol{\zeta}^{k+1}\right), \\
b\left(\mathbf{U}^{k+1}, q\right) & =0 \quad \forall q \in M\left(\boldsymbol{\zeta}^{k+1}\right),
\end{aligned}
$$

with

$$
\left\|\mathbf{A}_{k}\right\|_{L^{2}(\mathcal{O})^{2}} \leq C\left(\left|\boldsymbol{\zeta}\left(t_{k+1}\right)-\boldsymbol{\zeta}^{k+1}\right|+\Delta t+\sqrt{\Delta t}\left\|\frac{d^{2}}{d t^{2}}[\mathbf{u} \circ \widetilde{\boldsymbol{\psi}}]\right\|_{L^{2}\left(\mathcal{O} \times\left(t_{k}, t_{k+1}\right)\right)^{2}}\right) .
$$

Let us prove an approximation property of the function $\overline{\mathbf{X}}^{k}$ and we also recall an useful property on the change of variables which is given in [18].

Lemma 6.2. The functions $\overline{\mathbf{X}}^{k}, \widehat{\mathbf{X}}$ and $\widehat{\mathbf{J}}$ defined in (3.4), 6.2) and (6.3) respectively, satisfy the following estimates:

$$
\begin{aligned}
\left\|\widehat{\mathbf{X}}-\overline{\mathbf{X}}^{k}\right\|_{L^{2}(\mathcal{O})^{2}} \leq & C\left(\Delta t^{2}+\Delta t\left\|\mathbf{U}^{k}-\mathbf{u}^{k}\right\|_{L^{2}(\mathcal{O})^{2}}+\sqrt{\Delta t}\left\|\boldsymbol{\delta}_{k}\right\|_{L^{2}\left(\mathcal{O} \times\left(t_{k}, t_{k+1}\right)\right)^{2}}\right) \\
\|\widehat{\mathbf{J}}-\mathbf{I d}\|_{L^{2}(\mathcal{O})^{2}} \leq C\left(\Delta t^{2}+\Delta t\left\|\mathbf{U}^{k}-\mathbf{u}^{k}\right\|_{L^{2}(\mathcal{O})^{2}}\right. & +\sqrt{\Delta t}\left\|\boldsymbol{\delta}_{k}\right\|_{L^{2}\left(\mathcal{O} \times\left(t_{k}, t_{k+1}\right)\right)^{2}} \\
& \left.+\Delta t\left|\boldsymbol{\zeta}\left(t_{k}\right)-\boldsymbol{\zeta}^{k}\right|\right)
\end{aligned}
$$

with $\left\|\boldsymbol{\delta}_{k}\right\|_{L^{2}\left(\mathcal{O} \times\left(t_{k}, t_{k+1}\right)\right)^{2}} \leq C \Delta t\left\|\frac{\partial \mathbf{u}}{\partial t}\right\|_{L^{2}\left(\mathcal{O} \times\left(t_{k}, t_{k+1}\right)\right)^{2}}$ and $C$ a positive constant independent of $k$.

Proof. Let us define a new characteristic function $\overline{\bar{\psi}}$ associated with the semi-discretized velocity field as the solution of

$$
\left\{\begin{array}{l}
\frac{d}{d t} \overline{\overline{\boldsymbol{\psi}}}\left(t ; t_{k+1}, \mathbf{x}\right)=\mathbf{U}^{k}\left(\overline{\overline{\boldsymbol{\psi}}}\left(t ; t_{k+1}, \mathbf{x}\right)\right) \\
\overline{\overline{\boldsymbol{\psi}}}\left(t_{k+1} ; t_{k+1}, \mathbf{x}\right)=\mathbf{x}
\end{array}\right.
$$

and let us denote

$$
\overline{\overline{\mathbf{X}}}^{k}(\mathbf{x})=\overline{\overline{\boldsymbol{\psi}}}\left(t_{k} ; t_{k+1}, \mathbf{x}\right) \quad \forall \mathbf{x} \in \mathcal{O} .
$$

With a very slight modification of the proof of Lemma 6.5 from [18, we get

$$
\left\|\widehat{\mathbf{X}}-\overline{\overline{\mathbf{X}}}^{k}\right\|_{L^{2}(\mathcal{O})^{2}} \leq C\left(\Delta t^{2}+\sqrt{\Delta t}\left\|\boldsymbol{\delta}_{k}\right\|_{L^{2}\left(\mathcal{O} \times\left(t_{k}, t_{k+1}\right)\right)^{2}}\right) .
$$

The characteristic equations (3.3) and (6.9) can be written as follows:

$$
\begin{aligned}
& \overline{\boldsymbol{\psi}}\left(t ; t_{k+1}, \mathbf{x}\right)=\mathbf{x}-\mathbf{u}^{k}\left(\boldsymbol{\zeta}^{k}\right)\left(t-t_{k}\right)+\int_{t_{k+1}}^{t} \mathbf{u}^{k}\left(\overline{\boldsymbol{\psi}}\left(s ; t_{k+1}, \mathbf{x}\right)\right) \mathrm{d} s \\
& \overline{\overline{\boldsymbol{\psi}}}\left(t ; t_{k+1}, \mathbf{x}\right)=\mathbf{x}+\int_{t_{k+1}}^{t} \mathbf{U}^{k}\left(\overline{\overline{\boldsymbol{\psi}}}\left(s ; t_{k+1}, \mathbf{x}\right)\right) \mathrm{d} s
\end{aligned}
$$

for all $t \in\left[t_{k}, t_{k+1}\right]$. Subtracting the previous identities, we get

$$
\begin{aligned}
\overline{\boldsymbol{\psi}}\left(t ; t_{k+1}, \mathbf{x}\right)-\overline{\overline{\boldsymbol{\psi}}}\left(t ; t_{k+1}, \mathbf{x}\right)= & -\mathbf{u}^{k}\left(\boldsymbol{\zeta}^{k}\right)\left(t-t_{k}\right) \\
& +\int_{t_{k+1}}^{t}\left(\mathbf{u}^{k}\left(\overline{\boldsymbol{\psi}}\left(s ; t_{k+1}, \mathbf{x}\right)\right)-\mathbf{U}^{k}\left(\overline{\overline{\boldsymbol{\psi}}}\left(s ; t_{k+1}, \mathbf{x}\right)\right)\right) \mathrm{d} s .
\end{aligned}
$$


Taking the $L^{2}(\mathcal{O})^{2}$-norm, we obtain that

$$
\begin{aligned}
\left\|\overline{\boldsymbol{\psi}}\left(t ; t_{k+1}, \cdot\right)-\overline{\overline{\boldsymbol{\psi}}}\left(t ; t_{k+1}, \cdot\right)\right\|_{L^{2}(\mathcal{O})^{2}} & \leq C\left|\mathbf{u}^{k}\left(\boldsymbol{\zeta}^{k}\right)\right|\left(t-t_{k}\right) \\
& +\int_{t}^{t_{k+1}}\left\|\mathbf{u}^{k}\left(\overline{\boldsymbol{\psi}}\left(s ; t_{k+1}, \cdot\right)\right)-\mathbf{U}^{k}\left(\overline{\boldsymbol{\psi}}\left(s ; t_{k+1}, \cdot\right)\right)\right\|_{L^{2}(\mathcal{O})^{2}} \mathrm{~d} s \\
& +\int_{t}^{t_{k+1}}\left\|\mathbf{U}^{k}\left(\overline{\boldsymbol{\psi}}\left(s ; t_{k+1}, \cdot\right)\right)-\mathbf{U}^{k}\left(\overline{\overline{\boldsymbol{\psi}}}\left(s ; t_{k+1}, \cdot\right)\right)\right\|_{L^{2}(\mathcal{O})^{2}} \mathrm{~d} s .
\end{aligned}
$$

By using the property (5.7) and the regularity hypothesis 3.12 , we get

$$
\begin{aligned}
\left\|\overline{\boldsymbol{\psi}}\left(t ; t_{k+1}, \cdot\right)-\overline{\overline{\boldsymbol{\psi}}}\left(t ; t_{k+1}, \cdot\right)\right\|_{L^{2}(\mathcal{O})^{2}} \leq & C\left|\mathbf{u}^{k}\left(\boldsymbol{\zeta}^{k}\right)\right|\left(t-t_{k}\right)+\left\|\mathbf{u}^{k}-\mathbf{U}^{k}\right\|_{L^{2}(\mathcal{O})^{2}}\left(t_{k+1}-t\right) \\
& +\int_{t}^{t_{k+1}} C\left\|\overline{\boldsymbol{\psi}}\left(s ; t_{k+1}, \cdot\right)-\overline{\overline{\boldsymbol{\psi}}}\left(s ; t_{k+1}, \cdot\right)\right\|_{L^{2}(\mathcal{O})^{2}} \mathrm{~d} s .
\end{aligned}
$$

Then, due to Gronwall inequality, the above estimate yields

$$
\begin{aligned}
\left\|\overline{\boldsymbol{\psi}}\left(t ; t_{k+1}, \cdot\right)-\overline{\overline{\boldsymbol{\psi}}}\left(t ; t_{k+1}, \cdot\right)\right\|_{L^{2}(\mathcal{O})^{2}} \leq & C\left|\mathbf{u}^{k}\left(\boldsymbol{\zeta}^{k}\right)\right|\left(t-t_{k}\right)+\left\|\mathbf{u}^{k}-\mathbf{U}^{k}\right\|_{L^{2}(\mathcal{O})^{2}}\left(t_{k+1}-t\right) \\
& +C \Delta t^{2}\left(\left|\mathbf{u}^{k}\left(\boldsymbol{\zeta}^{k}\right)\right|+\left\|\mathbf{u}^{k}-\mathbf{U}^{k}\right\|_{L^{2}(\mathcal{O})^{2}}\right),
\end{aligned}
$$

for all $t \in\left[t_{k}, t_{k+1}\right]$. In particular, for $t=t_{k}$ we deduce that

$$
\begin{aligned}
\left\|\overline{\mathbf{X}}^{k}-\overline{\overline{\mathbf{X}}}^{k}\right\|_{L^{2}(\mathcal{O})^{2}} & \leq C \Delta t^{2}\left|\mathbf{u}^{k}\left(\boldsymbol{\zeta}^{k}\right)\right|+C \Delta t\left\|\mathbf{u}^{k}-\mathbf{U}^{k}\right\|_{L^{2}(\mathcal{O})^{2}} \\
& \leq C \Delta t^{2}\left|\mathbf{U}^{k}\left(\boldsymbol{\zeta}^{k}\right)\right|+C \Delta t^{2}\left|\mathbf{u}^{k}\left(\boldsymbol{\zeta}^{k}\right)-\mathbf{U}^{k}\left(\boldsymbol{\zeta}^{k}\right)\right|+C \Delta t\left\|\mathbf{u}^{k}-\mathbf{U}^{k}\right\|_{L^{2}(\mathcal{O})^{2}} .
\end{aligned}
$$

Combining the above inequality with (6.11) and using again the regularity hypothesis $(3.12$, we deduce the result 6.7 ).

The proof of 6.8 is done in [18, eq. (7.6)].

\subsection{Error estimate}

In this subsection, we give the proof of our first main result stated in Theorem 3.3 . To this end, let us subtract (3.7)-(3.8) from (6.4) $-(6.5)$ and we obtain

$$
\begin{gathered}
\frac{1}{\Delta t}\left(\rho^{k+1}\left(\mathbf{U}^{k+1}-\mathbf{u}^{k+1}\right), \boldsymbol{\varphi}\right)+a\left(\mathbf{U}^{k+1}-\mathbf{u}^{k+1}, \boldsymbol{\varphi}\right)+b\left(\boldsymbol{\varphi}, P^{k+1}-p^{k+1}\right) \\
=\frac{1}{\Delta t}\left(\rho^{k+1}\left(\widehat{\mathbf{J}}\left(\mathbf{U}^{k} \circ \widehat{\mathbf{X}}\right)-\mathbf{u}^{k} \circ \overline{\mathbf{X}}^{k}\right), \boldsymbol{\varphi}\right)+\left(\mathbf{A}_{k}, \boldsymbol{\varphi}\right) \quad \forall \boldsymbol{\varphi} \in \mathcal{K}\left(\boldsymbol{\zeta}^{k+1}\right), \\
b\left(\mathbf{U}^{k+1}-\mathbf{u}^{k+1}, q\right)=0 \quad \forall q \in M\left(\boldsymbol{\zeta}^{k+1}\right) .
\end{gathered}
$$

We choose the test functions $\boldsymbol{\varphi}=\mathbf{U}^{k+1}-\mathbf{u}^{k+1} \in \mathcal{K}\left(\boldsymbol{\zeta}^{k+1}\right)$ and $q=P^{k+1}-p^{k+1} \in$ $M\left(\boldsymbol{\zeta}^{k+1}\right)$ and we get that

$$
\begin{aligned}
\left(\rho ^ { k + 1 } \left(\mathbf{U}^{k+1}\right.\right. & \left.\left.-\mathbf{u}^{k+1}\right), \mathbf{U}^{k+1}-\mathbf{u}^{k+1}\right)+\Delta t a\left(\mathbf{U}^{k+1}-\mathbf{u}^{k+1}, \mathbf{U}^{k+1}-\mathbf{u}^{k+1}\right) \\
& =\left(\rho^{k+1}\left(\widehat{\mathbf{J}}\left(\mathbf{U}^{k} \circ \widehat{\mathbf{X}}\right)-\mathbf{u}^{k} \circ \overline{\mathbf{X}}^{k}\right), \mathbf{U}^{k+1}-\mathbf{u}^{k+1}\right)+\Delta t\left(\mathbf{A}_{k}, \mathbf{U}^{k+1}-\mathbf{u}^{k+1}\right),
\end{aligned}
$$

then due to Cauchy-Schwarz inequality, there exists a positive constant $C$ independent of $k$ such that

$$
\begin{aligned}
\left\|\sqrt{\rho^{k+1}}\left(\mathbf{U}^{k+1}-\mathbf{u}^{k+1}\right)\right\|_{L^{2}(\mathcal{O})^{2}} \leq\left\|\sqrt{\rho^{k+1}}\left(\widehat{\mathbf{J}}\left(\mathbf{U}^{k} \circ \widehat{\mathbf{X}}\right)-\mathbf{u}^{k} \circ \overline{\mathbf{X}}^{k}\right)\right\|_{L^{2}(\mathcal{O})^{2}} \\
+C \Delta t\left\|\mathbf{A}_{k}\right\|_{L^{2}(\mathcal{O})^{2}}
\end{aligned}
$$


In order to estimate the first term in the right hand side of $(6.12)$, we observe that

$$
\begin{aligned}
\sqrt{\rho^{k+1}}\left(\widehat{\mathbf{J}}\left(\mathbf{U}^{k} \circ \widehat{\mathbf{X}}\right)-\mathbf{u}^{k} \circ \overline{\mathbf{X}}^{k}\right) & =\sqrt{\rho^{k+1}}(\widehat{\mathbf{J}}-\mathbf{I} \mathbf{d})\left(\mathbf{U}^{k} \circ \widehat{\mathbf{X}}\right) \\
& +\sqrt{\rho^{k+1}}\left(\mathbf{U}^{k} \circ \widehat{\mathbf{X}}-\mathbf{U}^{k} \circ \overline{\mathbf{X}}^{k}\right) \\
& +\sqrt{\rho^{k+1}}\left(\mathbf{U}^{k}-\mathbf{u}^{k}\right) \circ \overline{\mathbf{X}}^{k},
\end{aligned}
$$

then using the regularity hypothesis $(3.12)$ and the definition of $\mathbf{U}^{k}$ given in $(6.1)$, we easily deduce

$$
\begin{aligned}
\left\|\sqrt{\rho^{k+1}}\left(\widehat{\mathbf{J}}\left(\mathbf{U}^{k} \circ \widehat{\mathbf{X}}\right)-\mathbf{u}^{k} \circ \overline{\mathbf{X}}^{k}\right)\right\|_{L^{2}(\mathcal{O})^{2}} \leq C\|\widehat{\mathbf{J}}-\mathbf{I d}\|_{L^{2}(\mathcal{O})^{2}} \\
+C\left\|\widehat{\mathbf{X}}-\overline{\mathbf{X}}^{k}\right\|_{L^{2}(\mathcal{O})^{2}}+\left\|\sqrt{\rho^{k+1}}\left(\mathbf{U}^{k}-\mathbf{u}^{k}\right) \circ \overline{\mathbf{X}}^{k}\right\|_{L^{2}(\mathcal{O})^{2}} .
\end{aligned}
$$

Then, by using the inequality 5.7 from Proposition 5.2 , we observe that

$$
\begin{aligned}
\left\|\sqrt{\rho^{k+1}}\left(\widehat{\mathbf{J}}\left(\mathbf{U}^{k} \circ \widehat{\mathbf{X}}\right)-\mathbf{u}^{k} \circ \overline{\mathbf{X}}^{k}\right)\right\|_{L^{2}(\mathcal{O})^{2}} & \leq C\|\widehat{\mathbf{J}}-\mathbf{I d}\|_{L^{2}(\mathcal{O})^{2}} \\
+ & +C\left\|\widehat{\mathbf{X}}-\overline{\mathbf{X}}^{k}\right\|_{L^{2}(\mathcal{O})^{2}}+\left\|\sqrt{\rho^{k}}\left(\mathbf{U}^{k}-\mathbf{u}^{k}\right)\right\|_{L^{2}(\mathcal{O})^{2}} .
\end{aligned}
$$

Due to the above estimate, the inequality $(6.12)$ becomes

$$
\begin{aligned}
\left\|\sqrt{\rho^{k+1}}\left(\mathbf{U}^{k+1}-\mathbf{u}^{k+1}\right)\right\|_{L^{2}(\mathcal{O})^{2}} \leq\left\|\sqrt{\rho^{k}}\left(\mathbf{U}^{k}-\mathbf{u}^{k}\right)\right\|_{L^{2}(\mathcal{O})^{2}}+C\|\widehat{\mathbf{J}}-\mathbf{I d}\|_{L^{2}(\mathcal{O})^{2}} \\
+C\left\|\widehat{\mathbf{X}}-\overline{\mathbf{X}}^{k}\right\|_{L^{2}(\mathcal{O})^{2}}+C \Delta t\left\|\mathbf{A}_{k}\right\|_{L^{2}(\mathcal{O})^{2}},
\end{aligned}
$$

then, taking into account the estimates $(6.6)$ from Proposition 6.1, $(6.7)-(6.8)$ from Lemma 6.2 we obtain

$$
\begin{aligned}
& \left\|\sqrt{\rho^{k+1}}\left(\mathbf{U}^{k+1}-\mathbf{u}^{k+1}\right)\right\|_{L^{2}(\mathcal{O})^{2}} \leq\left\|\sqrt{\rho^{k}}\left(\mathbf{U}^{k}-\mathbf{u}^{k}\right)\right\|_{L^{2}(\mathcal{O})^{2}} \\
& +C\left(\Delta t\left\|\mathbf{U}^{k}-\mathbf{u}^{k}\right\|_{L^{2}(\mathcal{O})^{2}}+\Delta t\left|\boldsymbol{\zeta}\left(t_{k}\right)-\boldsymbol{\zeta}^{k}\right|+\Delta t\left|\boldsymbol{\zeta}\left(t_{k+1}\right)-\boldsymbol{\zeta}^{k+1}\right|\right. \\
& \left.+\Delta t^{2}+\sqrt{\Delta t}\left\|\boldsymbol{\delta}_{k}\right\|_{L^{2}\left(\mathcal{O} \times\left(t_{k}, t_{k+1}\right)\right)^{2}}+(\Delta t)^{3 / 2}\left\|\frac{d^{2}}{d t^{2}}[\mathbf{u} \circ \widetilde{\boldsymbol{\psi}}]\right\|_{L^{2}\left(\mathcal{O} \times\left(t_{k}, t_{k+1}\right)\right)^{2}}\right) .
\end{aligned}
$$

Since

we get that

$$
\left\|\boldsymbol{\delta}_{k}\right\|_{L^{2}\left(\mathcal{O} \times\left(t_{k}, t_{k+1}\right)\right)^{2}} \leq C \Delta t\left\|\frac{\partial \mathbf{u}}{\partial t}\right\|_{L^{2}\left(\mathcal{O} \times\left(t_{k}, t_{k+1}\right)\right)^{2}},
$$

$$
\begin{aligned}
& \left\|\sqrt{\rho^{k+1}}\left(\mathbf{U}^{k+1}-\mathbf{u}^{k+1}\right)\right\|_{L^{2}(\mathcal{O})^{2}} \leq\left\|\sqrt{\rho^{k}}\left(\mathbf{U}^{k}-\mathbf{u}^{k}\right)\right\|_{L^{2}(\mathcal{O})^{2}} \\
& \quad+C\left(\Delta t\left\|\mathbf{U}^{k}-\mathbf{u}^{k}\right\|_{L^{2}(\mathcal{O})^{2}}+\Delta t\left|\boldsymbol{\zeta}\left(t_{k}\right)-\boldsymbol{\zeta}^{k}\right|+\Delta t\left|\boldsymbol{\zeta}\left(t_{k+1}\right)-\boldsymbol{\zeta}^{k+1}\right|\right. \\
& \left.\quad+\Delta t^{2}+(\Delta t)^{3 / 2}\left\|\frac{\partial \mathbf{u}}{\partial t}\right\|_{L^{2}\left(\mathcal{O} \times\left(t_{k}, t_{k+1}\right)\right)^{2}}+(\Delta t)^{3 / 2}\left\|\frac{d^{2}}{d t^{2}}[\mathbf{u} \circ \widetilde{\boldsymbol{\psi}}]\right\|_{L^{2}\left(\mathcal{O} \times\left(t_{k}, t_{k+1}\right)\right)^{2}}\right) .
\end{aligned}
$$

Let us also recall (see [18, Eq.(7.9)]) that

$$
\left|\boldsymbol{\zeta}\left(t_{k+1}\right)-\boldsymbol{\zeta}^{k+1}\right| \leq\left|\boldsymbol{\zeta}\left(t_{k}\right)-\boldsymbol{\zeta}^{k}\right|+C \Delta t\left\|\mathbf{U}^{k}-\mathbf{u}^{k}\right\|_{L^{2}(\mathcal{O})^{2}}+C \Delta t^{2},
$$

then, the estimate (6.15) yield

$$
\begin{aligned}
& \left\|\sqrt{\rho^{k+1}}\left(\mathbf{U}^{k+1}-\mathbf{u}^{k+1}\right)\right\|_{L^{2}(\mathcal{O})^{2}}+\left|\boldsymbol{\zeta}\left(t_{k+1}\right)-\boldsymbol{\zeta}^{k+1}\right| \\
& \leq(1+C \Delta t)\left(\left\|\sqrt{\rho^{k}}\left(\mathbf{U}^{k}-\mathbf{u}^{k}\right)\right\|_{L^{2}(\mathcal{O})^{2}}+\left|\boldsymbol{\zeta}\left(t_{k}\right)-\boldsymbol{\zeta}^{k}\right|\right) \\
& +C\left(\Delta t^{2}+(\Delta t)^{3 / 2}\left\|\frac{\partial \mathbf{u}}{\partial t}\right\|_{L^{2}\left(\mathcal{O} \times\left(t_{k}, t_{k+1}\right)\right)^{2}}+(\Delta t)^{3 / 2}\left\|\frac{d^{2}}{d t^{2}}[\mathbf{u} \circ \widetilde{\boldsymbol{\psi}}]\right\|_{L^{2}\left(\mathcal{O} \times\left(t_{k}, t_{k+1}\right)\right)^{2}}\right) .
\end{aligned}
$$


For any $k \in\{0, \ldots, N\}$, let us define

$$
E^{k}=\left\|\sqrt{\rho^{k}}\left(\mathbf{U}^{k}-\mathbf{u}^{k}\right)\right\|_{L^{2}(\mathcal{O})^{2}}+\left|\boldsymbol{\zeta}\left(t_{k}\right)-\boldsymbol{\zeta}^{k}\right|,
$$

then according to the estimate 6.17 we write

$$
\begin{aligned}
E^{k+1} \leq(1+C \Delta t) E^{k}+C\left(\Delta t^{2}+(\Delta t)^{3 / 2}\left\|\frac{\partial \mathbf{u}}{\partial t}\right\|_{L^{2}\left(\mathcal{O} \times\left(t_{k}, t_{k+1}\right)\right)^{2}}\right. & \\
& \left.+(\Delta t)^{3 / 2}\left\|\frac{d^{2}}{d t^{2}}[\mathbf{u} \circ \widetilde{\boldsymbol{\psi}}]\right\|_{L^{2}\left(\mathcal{O} \times\left(t_{k}, t_{k+1}\right)\right)^{2}}\right) .
\end{aligned}
$$

By applying the discrete Gronwall Lemma, we deduce that for any $k \in\{0, \ldots, N\}$,

$$
E^{k} \leq C\left(\Delta t+(\Delta t)^{3 / 2}\left\|\frac{\partial \mathbf{u}}{\partial t}\right\|_{L^{2}(\mathcal{O} \times(0, T))^{2}}+(\Delta t)^{3 / 2}\left\|\frac{d^{2}}{d t^{2}}[\mathbf{u} \circ \widetilde{\boldsymbol{\psi}}]\right\|_{L^{2}(\mathcal{O} \times(0, T))^{2}}\right),
$$

then using the hypothesis 3.12 we get

$$
\left\|\mathbf{U}^{k}-\mathbf{u}^{k}\right\|_{L^{2}(\mathcal{O})^{2}}+\left|\boldsymbol{\zeta}\left(t_{k}\right)-\boldsymbol{\zeta}^{k}\right| \leq C \Delta t .
$$

The above estimate together with the definition of $\mathbf{U}^{k}$ from (6.1) and properties on the change of variables $\mathbf{X}^{k}$ given in [18, Lemmas 5.5-5.6], we conclude the proof of 3.15 from our first main result given in Theorem 3.3 .

\section{Proof of the second main result}

Let us give the proof of the second main result stated in Theorem 4.1 which concern the convergence of the fully discretization scheme (4.7)-4.8. To this end, we first introduce the transformed system (7.4)-7.5 below and we prove some important estimates on the transform velocity field. Then we prove the second main result.

\subsection{Preliminaries}

Since we need to compare the exact solution $\mathbf{u}\left(t_{k}\right) \in \mathcal{K}\left(\boldsymbol{\zeta}\left(t_{k}\right)\right)$, which is a rigid velocity field in $B\left(\boldsymbol{\zeta}\left(t_{k}\right)\right)$ with $\mathbf{u}_{h}^{k} \in \mathcal{K}\left(\boldsymbol{\zeta}_{h}^{k}\right)$ which is a rigid velocity field in $B\left(\boldsymbol{\zeta}_{h}^{k}\right)$, we are going to use the change of variable $X_{\boldsymbol{\zeta}_{1}, \boldsymbol{\zeta}_{2}}$ which maps the ball $B\left(\boldsymbol{\zeta}_{1}\right)$ into the ball $B\left(\boldsymbol{\zeta}_{2}\right)$. We define

$$
\mathbf{X}_{h}^{k}=\mathbf{X}_{\zeta_{h}^{k}, \boldsymbol{\zeta}\left(t_{k}\right)}, \quad \mathbf{Y}_{h}^{k}=\mathbf{Y}_{\boldsymbol{\zeta}_{h}^{k}, \boldsymbol{\zeta}\left(t_{k}\right)},
$$

where $Y_{\boldsymbol{\zeta}_{1}, \boldsymbol{\zeta}_{2}}$ is the inverse mapping of $X_{\boldsymbol{\zeta}_{1}, \boldsymbol{\zeta}_{2}}$. We also define

$$
\mathbf{U}_{h}^{k}(\mathbf{y})=\mathbf{J}_{\mathbf{Y}_{h}^{k}}\left(\mathbf{X}_{h}^{k}(\mathbf{y})\right) \mathbf{u}\left(\mathbf{X}_{h}^{k}(\mathbf{y}), t_{k}\right), \quad P_{h}^{k}(\mathbf{y})=p\left(\mathbf{X}_{h}^{k}(\mathbf{y}), t_{k}\right)
$$

where $\mathbf{J}_{\mathbf{Y}_{h}^{k}}$ is the determinant of the jacobian matrix of $\mathbf{Y}_{h}^{k}$. We recall that $\mathbf{U}_{h}^{k} \in \widehat{\mathcal{K}}\left(\boldsymbol{\zeta}_{h}^{k}\right)$ and $P_{h}^{k} \in M\left(\boldsymbol{\zeta}_{h}^{k}\right)$. Let us introduce the following notations that will be useful in the sequel:

$$
\widehat{\mathbf{X}}_{h}=\mathbf{Y}_{h}^{k} \circ \widetilde{\mathbf{X}} \circ \mathbf{X}_{h}^{k+1}
$$

and

$$
\widehat{\mathbf{J}}_{h}=\left(\mathbf{J}_{\mathbf{Y}_{h}^{k+1}} \circ \mathbf{X}_{h}^{k+1}\right)\left(\mathbf{J}_{\mathbf{X}_{h}^{k}} \circ \widehat{\mathbf{X}}_{h}\right) .
$$

We observe that the characteristics functions satisfy the properties depicted on the following diagram:

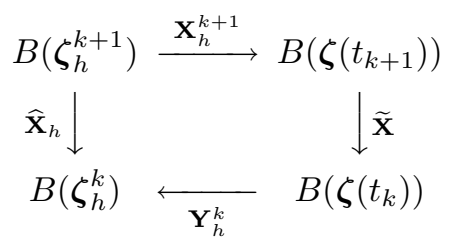


The transformed functions $\mathbf{U}_{h}^{k+1}$ and $P_{h}^{k+1}$ satisfy a mixed weak formulation with test functions in $\mathcal{K}\left(\boldsymbol{\zeta}_{h}^{k+1}\right)$ and $M\left(\boldsymbol{\zeta}_{h}^{k+1}\right)$.

Proposition 7.1. The functions $\left(\mathbf{U}_{h}^{k+1}, P_{h}^{k+1}\right)$ defined by 7.1 satisfy

$$
\begin{aligned}
& \frac{1}{\Delta t}\left(\rho_{h}^{k+1}\left[\mathbf{U}_{h}^{k+1}-\widehat{\mathbf{J}}_{h}\left(\mathbf{U}_{h}^{k} \circ \widehat{\mathbf{X}}_{h}\right)\right], \boldsymbol{\varphi}\right)+a\left(\mathbf{U}_{h}^{k+1}, \boldsymbol{\varphi}\right)+b\left(\boldsymbol{\varphi}, P_{h}^{k+1}\right) \\
&=\left(\rho_{h}^{k+1} \mathbf{f}_{h}^{k+1}, \boldsymbol{\varphi}\right)+\left(\mathbf{A}_{h}^{k}, \boldsymbol{\varphi}\right) \quad \forall \boldsymbol{\varphi} \in \mathcal{K}\left(\boldsymbol{\zeta}_{h}^{k+1}\right), \\
& b\left(\mathbf{U}_{h}^{k+1}, q\right)=0 \quad \forall q \in M\left(\boldsymbol{\zeta}_{h}^{k+1}\right),
\end{aligned}
$$

with

$$
\left\|\mathbf{A}_{h}^{k}\right\|_{L^{2}(\mathcal{O})^{2}} \leq C\left(\left|\boldsymbol{\zeta}\left(t_{k+1}\right)-\boldsymbol{\zeta}_{h}^{k+1}\right|+h+\Delta t+\sqrt{\Delta t}\left\|\frac{d^{2}}{d t^{2}}[\mathbf{u} \circ \widetilde{\boldsymbol{\psi}}]\right\|_{L^{2}\left(\mathcal{O} \times\left(t_{k}, t_{k+1}\right)\right)^{2}}\right) .
$$

The above result can be obtained as in [18, Proposition 6.2] with a very slight modification of the proof.

In the following lemma, we state an important result on the transformed velocity field. Precisely, we prove the existence of a velocity field $\mathbf{U}_{h, e x t}^{k}$ near $\mathbf{U}_{h}^{k}$ which is rigid in a $h$ neighbourhood of the ball $B\left(\boldsymbol{\zeta}_{h}^{k}\right)$. Moreover, this function is a rigid velocity field in $Q_{h}$.

Lemma 7.2. For any $k \in\{0, \ldots, N\}$ and $h \in(0,1)$, there exists a velocity field $\mathbf{U}_{h, \text { ext }}^{k} \in$ $H_{0}^{1}(\mathcal{O})^{2}$ such that

$$
\begin{gathered}
\mathbf{U}_{h, e x t}^{k}(\mathbf{x})=\mathbf{U}_{h}^{k}(\mathbf{x}) \quad \forall \mathbf{x} \in B\left(\boldsymbol{\zeta}_{h}^{k}\right), \\
\mathrm{D}\left(\mathbf{U}_{h, e x t}^{k}\right)=0 \text { in }\left\{\mathbf{x} \in \mathcal{O}:\left|\mathbf{x}-\boldsymbol{\zeta}_{h}^{k}\right|<1+h\right\}, \\
\left\|\mathbf{U}_{h}^{k}-\mathbf{U}_{h, e x t}^{k}\right\|_{L^{2}(\mathcal{O})^{2}} \leq C h^{3 / 2}, \\
\left\|\mathbf{U}_{h, \text { ext }}^{k}\right\|_{H^{1}(\mathcal{O})^{2}} \leq C,
\end{gathered}
$$

where $C$ is a positive constant independent of $h$ and $k$.

Proof. Since $\mathbf{U}_{h}^{k} \in H_{0}^{1}(\mathcal{O})^{2}$ and $\operatorname{div} \mathbf{U}_{h}^{k}=0$, there exists a stream function $\Phi \in H^{2}(\mathcal{O}) \cap$ $H_{0}^{1}(\mathcal{O})$ such that $\mathbf{U}_{h}^{k}=\nabla^{\perp} \Phi$.

It clearly suffices to prove that there exists a stream function $\Phi_{\text {ext }} \in H^{2}(\mathcal{O}) \cap H_{0}^{1}(\mathcal{O})$, such that $\nabla^{\perp} \Phi_{\text {ext }}$ satisfies the conditions $(7.7)-(7.10)$.

To this end, let us observe that since $\mathrm{D}\left(\mathbf{U}_{h}^{k}\right)=0$ in $B\left(\boldsymbol{\zeta}_{h}^{k}\right)$, there exist some constants $a, c \in \mathbb{R}$ and $\mathbf{b} \in \mathbb{R}^{2}$ such that

$$
\Phi(\mathbf{x})=a+\mathbf{b} \cdot \mathbf{x}+c|\mathbf{x}|^{2} \quad \forall \mathbf{x} \in B\left(\boldsymbol{\zeta}_{h}^{k}\right) .
$$

We denote

$$
w(\mathbf{x})=\Phi(\mathbf{x})-\left(a+\mathbf{b} \cdot \mathbf{x}+c|\mathbf{x}|^{2}\right) \quad \forall \mathbf{x} \in \mathcal{O},
$$

then it is clear that

$$
w(\mathbf{x})=0 \quad \forall \mathbf{x} \in B\left(\boldsymbol{\zeta}_{h}^{k}\right) .
$$

Let us define the stream function $\Phi_{e x t}$ as follows:

$$
\Phi_{e x t}(\mathbf{x})=\Phi(\mathbf{x})-w(\mathbf{x}) \rho\left(\left|\mathbf{x}-\boldsymbol{\zeta}_{h}^{k}\right|\right) \quad \forall \mathbf{x} \in \mathcal{O},
$$

where the real function $\rho \in H^{2}(\mathbb{R})$ is given by the following formula

$$
\rho(s)= \begin{cases}1 & \text { if } s<1+h, \\ \frac{1}{2}\left(\cos \left(\frac{s-(1+h)}{h} \pi\right)+1\right) & \text { if } 1+h \leq s \leq 1+2 h, \\ 0 & \text { if } 1+2 h<s\end{cases}
$$

Using this definition, one can easily check that $\Phi_{\text {ext }}(\mathbf{x})=\Phi(\mathbf{x})$ for all $\mathbf{x}$ such that $\mid \mathbf{x}-$ $\boldsymbol{\zeta}_{h}^{k} \mid \leq 1$ or $\left|\mathbf{x}-\zeta_{h}^{k}\right| \geq 1+2 h$. Then $\nabla^{\perp} \Phi_{e x t} \in H_{0}^{1}(\mathcal{O})^{2}$ and satisfies the identity (7.7). 
Additionally, if $\left|\mathbf{x}-\boldsymbol{\zeta}_{h}^{k}\right|<1+h$, we have $\Phi_{\text {ext }}(\mathbf{x})=a+\mathbf{b} \cdot \mathbf{x}+c|\mathbf{x}|^{2}$ and this identity implies that $\nabla^{\perp} \Phi_{\text {ext }}$ satisfies $(7.8)$.

In order to prove that $\nabla^{\perp} \Phi_{\text {ext }}$ satisfies the estimates 7.9 and 7.10 , we first remark that

$$
\left(\Phi-\Phi_{\text {ext }}\right)(\mathbf{x})=0 \quad \forall \mathbf{x} \in \mathcal{O} \backslash \mathcal{A}_{1,1+2 h},
$$

where we have denoted by $\mathcal{A}_{1,1+2 h}$ the annulus enclosed between the circles of radius 1 , respectively $1+2 h$ and with center at $\boldsymbol{\zeta}_{h}^{k}$.

Let us now differentiate the identity $(7.13)$ and for any $i, j \in\{1,2\}$, we get that

$$
\frac{\partial\left(\Phi-\Phi_{e x t}\right)}{\partial x_{i}}=\frac{\partial w}{\partial x_{i}} \rho+w \rho^{\prime} \frac{x_{i}-\zeta_{h, i}^{k}}{\left|\mathbf{x}-\zeta_{h}^{k}\right|}
$$

and

$$
\begin{aligned}
\frac{\partial^{2}\left(\Phi-\Phi_{e x t}\right)}{\partial x_{i} \partial x_{j}} & =\frac{\partial^{2} w}{\partial x_{i} \partial x_{j}} \rho+\frac{\partial w}{\partial x_{i}} \rho^{\prime} \frac{x_{j}-\zeta_{h, j}^{k}}{\left|\mathbf{x}-\zeta_{h}^{k}\right|}+\frac{\partial w}{\partial x_{j}} \rho^{\prime} \frac{x_{i}-\zeta_{h, i}^{k}}{\left|\mathbf{x}-\zeta_{h}^{k}\right|} \\
+w \rho^{\prime \prime} & \frac{\left(x_{i}-\zeta_{h, i}^{k}\right)\left(x_{j}-\zeta_{h, j}^{k}\right)}{\left|\mathbf{x}-\boldsymbol{\zeta}_{h}^{k}\right|^{2}}+w \rho^{\prime}\left(\frac{\delta_{i j}}{\left|\mathbf{x}-\boldsymbol{\zeta}_{h}^{k}\right|}-\frac{\left(x_{i}-\zeta_{h, i}^{k}\right)\left(x_{j}-\zeta_{h, j}^{k}\right)}{\left|\mathbf{x}-\boldsymbol{\zeta}_{h}^{k}\right|^{3}}\right) .
\end{aligned}
$$

Taking the $L^{2}(\mathcal{O})$-norm in the estimates $7.15-(7.16)$, using the identity $(7.14)$ and the properties $|\rho| \leq 1,\left|\rho^{\prime}\right| \leq 2 / h$ and $\left|\rho^{\prime \prime}\right| \leq 5 / h^{2}$, we obtain that for all $i, j \in\{1,2\}$,

$$
\left\|\frac{\partial\left(\Phi-\Phi_{e x t}\right)}{\partial x_{i}}\right\|_{L^{2}(\mathcal{O})} \leq\left\|\frac{\partial w}{\partial x_{i}}\right\|_{L^{2}\left(\mathcal{A}_{1,1+2 h}\right)}+\frac{2}{h}\|w\|_{L^{2}\left(\mathcal{A}_{1,1+2 h}\right)}
$$

and

$$
\begin{aligned}
\left\|\frac{\partial^{2}\left(\Phi-\Phi_{e x t}\right)}{\partial x_{i} \partial x_{j}}\right\|_{L^{2}(\mathcal{O})} & \leq\left\|\frac{\partial^{2} w}{\partial x_{i} \partial x_{j}}\right\|_{L^{2}\left(\mathcal{A}_{1,1+2 h}\right)}+\frac{2}{h}\left\|\frac{\partial w}{\partial x_{i}}\right\|_{L^{2}\left(\mathcal{A}_{1,1+2 h}\right)} \\
+ & \frac{2}{h}\left\|\frac{\partial w}{\partial x_{j}}\right\|_{L^{2}\left(\mathcal{A}_{1,1+2 h}\right)}+\frac{5}{h^{2}}\|w\|_{L^{2}\left(\mathcal{A}_{1,1+2 h}\right)}+\frac{4}{h}\|w\|_{L^{2}\left(\mathcal{A}_{1,1+2 h}\right)} .
\end{aligned}
$$

In order to finish the proof, we need to estimate the different norms of $w$ on the annulus $\mathcal{A}_{1,1+2 h}$. To this end, let us take an arbitrary $\mathbf{x} \in \mathcal{A}_{1,1+2 h}$. It is easy to see that there exists $\mathbf{y} \in B\left(\boldsymbol{\zeta}_{h}^{k}\right)$ such that $|\mathbf{x}-\mathbf{y}| \leq 2 h$. Since $\nabla^{\perp} \Phi$ is a Lipschitz function (see the hypothesis (3.12) and the definition (7.1) ), we get that $\nabla w$ is also a Lipschitz function, with the Lipschitz constant $L$ independent of $h$. Using this property and $(7.12)$, we have

$$
|\nabla w(\mathbf{x})|=|\nabla w(\mathbf{x})-\nabla w(\mathbf{y})| \leq L|\mathbf{x}-\mathbf{y}| \leq 2 L h
$$

and

$$
\begin{aligned}
|w(\mathbf{x})| & =|w(\mathbf{x})-w(\mathbf{y})| \leq|\nabla w(\mathbf{y}+\lambda(\mathbf{x}-\mathbf{y}))| \cdot|\mathbf{x}-\mathbf{y}| \\
& =|\nabla w(\mathbf{y}+\lambda(\mathbf{x}-\mathbf{y}))-\nabla w(\mathbf{y})| \cdot|\mathbf{x}-\mathbf{y}| \leq L \lambda|\mathbf{x}-\mathbf{y}|^{2} \leq 4 \lambda L h^{2},
\end{aligned}
$$

for some $\lambda \in(0,1)$. Taking the $L^{2}\left(\mathcal{A}_{1,1+2 h}\right)$-norm in the estimates 7.19$)$ and $(7.20)$, we deduce

$$
\begin{aligned}
\|w\|_{L^{2}\left(\mathcal{A}_{1,1+2 h}\right)} & \leq C h^{5 / 2}, \\
\|\nabla w\|_{L^{2}\left(\mathcal{A}_{1,1+2 h}\right)^{2}} & \leq C h^{3 / 2} .
\end{aligned}
$$

Combining (7.17) with 7.21 -7.22 , we obtain the inequality 7.9). Moreover, the estimate (7.10) is a direct consequence of (7.18), (7.21)- 7.22 and the fact that $\|w\|_{H^{2}(\mathcal{O})}$ is independent of $h$ (see the definition of $w$ given in (7.11)). Thus, we conclude the proof of Lemma 7.2 . 
Using the above lemma, let us prove the following crucial estimate:

Proposition 7.3. For any $k \in\{0,1, \ldots, N\}$ and $h \in(0,1)$, the following estimate holds:

$$
\left\|\mathbf{U}_{h}^{k}-\mathbf{P}\left(\boldsymbol{\zeta}_{h}^{k}\right) \mathbf{U}_{h}^{k}\right\|_{L^{2}(\mathcal{O})^{2}} \leq C h,
$$

where $C$ is a positive constant independent of $h$.

Proof. Using Lemma 7.2 there exists $\mathbf{U}_{h, \text { ext }}^{k} \in H_{0}^{1}(\mathcal{O})^{2}$ satisfying $(7.7)-(7.10)$. We can write

$$
\begin{aligned}
\left\|\mathbf{U}_{h}^{k}-\mathbf{P}\left(\boldsymbol{\zeta}_{h}^{k}\right) \mathbf{U}_{h}^{k}\right\|_{L^{2}(\mathcal{O})^{2}} \leq\left\|\mathbf{U}_{h}^{k}-\mathbf{U}_{h, e x t}^{k}\right\|_{L^{2}(\mathcal{O})^{2}} & +\left\|\mathbf{U}_{h, e x t}^{k}-\mathbf{P}\left(\boldsymbol{\zeta}_{h}^{k}\right) \mathbf{U}_{h, e x t}^{k}\right\|_{L^{2}(\mathcal{O})^{2}} \\
& +\left\|\mathbf{P}\left(\boldsymbol{\zeta}_{h}^{k}\right) \mathbf{U}_{h, e x t}^{k}-\mathbf{P}\left(\boldsymbol{\zeta}_{h}^{k}\right) \mathbf{U}_{h}^{k}\right\|_{L^{2}(\mathcal{O})^{2}}
\end{aligned}
$$

then, since $\mathbf{P}\left(\boldsymbol{\zeta}_{h}^{k}\right)$ is an orthogonal projection from $L^{2}(\mathcal{O})^{2}$ onto $\mathcal{R}_{h}\left(\boldsymbol{\zeta}_{h}^{k}\right)$, we get

$$
\left\|\mathbf{U}_{h}^{k}-\mathbf{P}\left(\boldsymbol{\zeta}_{h}^{k}\right) \mathbf{U}_{h}^{k}\right\|_{L^{2}(\mathcal{O})^{2}} \leq 2\left\|\mathbf{U}_{h}^{k}-\mathbf{U}_{h, e x t}^{k}\right\|_{L^{2}(\mathcal{O})^{2}}+\left\|\mathbf{U}_{h, e x t}^{k}-\mathbf{P}\left(\boldsymbol{\zeta}_{h}^{k}\right) \mathbf{U}_{h, e x t}^{k}\right\|_{L^{2}(\mathcal{O})^{2}} .
$$

Let $\Phi_{\text {ext }}$ be the stream function corresponding to $\mathbf{U}_{h, \text { ext }}^{k}$ and $\Phi_{I}$ be the $\mathbb{P}_{2}$-Lagrange interpolated function of $\Phi_{\text {ext }}$ on the triangulation $\mathcal{T}_{h}$. Since $\mathbf{U}_{h, \text { ext }}^{k}$ is a rigid velocity field on $Q_{h}$, the function $\Phi_{e x t}$ is quadratic on $Q_{h}$ and thus

$$
\Phi_{I}(\mathbf{x})=\Phi_{\text {ext }}(\mathbf{x}) \quad \forall \mathbf{x} \in Q_{h}
$$

and $\nabla^{\perp} \Phi_{I}$ is a rigid velocity field in $Q_{h}$. This implies that $\nabla^{\perp} \Phi_{I} \in \mathcal{R}_{h}\left(\zeta_{h}^{k}\right)$.

By using the definition of the orthogonal projection and due to the classical estimates of the interpolated functions (see, for instance, [6, Lemma A.2, p. 99]), we deduce that

$$
\begin{aligned}
\left\|\mathbf{U}_{h, e x t}^{k}-\mathbf{P}\left(\boldsymbol{\zeta}_{h}^{k}\right) \mathbf{U}_{h, e x t}^{k}\right\|_{L^{2}(\mathcal{O})^{2}} & \leq\left\|\mathbf{U}_{h, e x t}^{k}-\nabla^{\perp} \Phi_{I}\right\|_{L^{2}(\mathcal{O})^{2}}=\left\|\nabla^{\perp} \Phi_{e x t}-\nabla^{\perp} \Phi_{I}\right\|_{L^{2}(\mathcal{O})^{2}} \\
& \leq C h\left\|\Phi_{e x t}\right\|_{H^{2}(\mathcal{O})^{2}} \leq C h\left\|\mathbf{U}_{h, e x t}^{k}\right\|_{H^{1}(\mathcal{O})^{2}} .
\end{aligned}
$$

Let us now conclude the proof of our result by noting that the estimate 7.23 is a direct consequence of the inequality 7.24 combined with $(7.25)$ and the estimates $7.94-7.10$ from Lemma 7.2 .

Let us now state an important estimate on the $L^{2}(\mathcal{O})$-norm of the difference between the density functions $\rho_{h}^{k}$ and $\bar{\rho}_{h}^{k}$ defined in 4.5 and 4.6 , respectively.

Lemma 7.4. There exists a positive constant $C$, independent of $h$ and $k$, such that

$$
\left\|\rho_{h}^{k}-\bar{\rho}_{h}^{k}\right\|_{L^{2}(\mathcal{O})} \leq C \sqrt{h} .
$$

Proof. Using the definitions of $\rho_{h}^{k}$ and $\bar{\rho}_{h}^{k}$ given in 4.5 and (4.6) respectively, we have that

$$
\rho_{h}^{k}-\bar{\rho}_{h}^{k}=0 \quad \text { in } \mathcal{F}_{4} \cup B\left(\boldsymbol{\zeta}_{h}^{k}\right) .
$$

Taking the $L^{2}(\mathcal{O})$-norm, we deduce that

$$
\left\|\rho_{h}^{k}-\bar{\rho}_{h}^{k}\right\|_{L^{2}(\mathcal{O})}^{2}=\int_{\mathcal{F}_{2} \cup \mathcal{F}_{3}}\left|\rho_{h}^{k}(\mathbf{x})-\bar{\rho}_{h}^{k}(\mathbf{x})\right|^{2} d \mathbf{x} \leq\left|\rho_{f}-\rho_{s}\right|^{2} \operatorname{mes}\left(\mathcal{F}_{2} \cup \mathcal{F}_{3}\right) .
$$

The region $\mathcal{F}_{2} \cup \mathcal{F}_{3}$ is contained into the annulus of center $\boldsymbol{\zeta}_{h}^{k}$ with radius $r_{1}=1-h$ and $r_{2}=1+2 h$ (see Figure 1). Then, the area of the region $\mathcal{F}_{2} \cup \mathcal{F}_{3}$ can be estimated as follows:

$$
\operatorname{mes}\left(\mathcal{F}_{2} \cup \mathcal{F}_{3}\right) \leq \pi\left((1+2 h)^{2}-(1-h)^{2}\right)=3 \pi h(h+2) .
$$

Combining this estimate with 7.27 , we conclude that the estimate 7.26 holds. 
Let us now prove approximation properties of the characteristic function defined in 4.2 and also properties on the change of variables.

Lemma 7.5. The functions $\overline{\mathbf{X}}_{h}^{k}, \widehat{\mathbf{X}}_{h}$ and $\widehat{\mathbf{J}}_{h}$ defined by (4.3), (7.2) and (7.3) respectively, satisfy the following estimates:

$$
\begin{gathered}
\left\|\widehat{\mathbf{X}}_{h}-\overline{\mathbf{X}}_{h}^{k}\right\|_{L^{2}(\mathcal{O})^{2}} \leq C\left(\Delta t^{2}+h \Delta t+\Delta t\left\|\mathbf{U}_{h}^{k}-\mathbf{u}_{h}^{k}\right\|_{L^{2}(\mathcal{O})^{2}}\right. \\
\left.+\sqrt{\Delta t}\left\|\boldsymbol{\delta}_{k}\right\|_{L^{2}\left(\mathcal{O} \times\left(t_{k}, t_{k+1}\right)\right)^{2}}\right) \\
\left\|\widehat{\mathbf{J}}_{h}-\mathbf{I d}\right\|_{L^{2}(\mathcal{O})^{2}} \leq C\left(\Delta t^{2}+\Delta t\left\|\mathbf{U}_{h}^{k}-\mathbf{u}_{h}^{k}\right\|_{L^{2}(\mathcal{O})^{2}}+\sqrt{\Delta t}\left\|\boldsymbol{\delta}_{k}\right\|_{L^{2}\left(\mathcal{O} \times\left(t_{k}, t_{k+1}\right)\right)^{2}}\right. \\
\left.+\Delta t\left|\boldsymbol{\zeta}\left(t_{k}\right)-\boldsymbol{\zeta}_{h}^{k}\right|\right)
\end{gathered}
$$

with

$$
\left\|\boldsymbol{\delta}_{k}\right\|_{L^{2}\left(\mathcal{O} \times\left(t_{k}, t_{k+1}\right)\right)^{2}} \leq C \Delta t\left\|\frac{\partial \mathbf{u}}{\partial t}\right\|_{L^{2}\left(\mathcal{O} \times\left(t_{k}, t_{k+1}\right)\right)^{2}} .
$$

Proof. Analogous to the proof of Lemma 6.2, we define the characteristic function $\overline{\overline{\boldsymbol{\psi}}}_{h}^{k}$ associated with the fully-discrete velocity field as the solution of

$$
\left\{\begin{array}{l}
\frac{d}{d t} \overline{\overline{\boldsymbol{\psi}}}_{h}^{k}\left(t ; t_{k+1}, \mathbf{x}\right)=\mathbf{U}_{h}^{k}\left(\overline{\overline{\boldsymbol{\psi}}}_{h}^{k}\left(t ; t_{k+1}, \mathbf{x}\right)\right) \\
\overline{\overline{\boldsymbol{\psi}}}_{h}^{k}\left(t_{k+1} ; t_{k+1}, \mathbf{x}\right)=\mathbf{x}
\end{array}\right.
$$

and we denote

$$
\overline{\overline{\mathbf{X}}}_{h}^{k}(\mathbf{x})=\overline{\overline{\boldsymbol{\psi}}}_{h}^{k}\left(t_{k} ; t_{k+1}, \mathbf{x}\right) \quad \forall \mathbf{x} \in \mathcal{O} .
$$

With a very slight modification of the proof of Lemma 6.5 in [18, we get

$$
\left\|\widehat{\mathbf{X}}_{h}-\overline{\overline{\mathbf{X}}}_{h}^{k}\right\|_{L^{2}(\mathcal{O})^{2}} \leq C\left(\Delta t^{2}+\sqrt{\Delta t}\left\|\boldsymbol{\delta}_{k}\right\|_{L^{2}\left(\mathcal{O} \times\left(t_{k}, t_{k+1}\right)\right)^{2}}\right) .
$$

Let us observe that the characteristic equations 4.2 and 7.31 can be written as follows: for any $t \in\left[t_{k}, t_{k+1}\right]$, we have

$$
\begin{aligned}
\overline{\boldsymbol{\psi}}_{h}^{k}\left(t ; t_{k+1}, \mathbf{x}\right)= & \mathbf{x}-\mathbf{u}_{h}^{k}\left(\boldsymbol{\zeta}_{h}^{k}\right) \Delta t+\int_{t_{k+1}}^{t} \mathbf{P}\left(\boldsymbol{\zeta}_{h}^{k}\right) \mathbf{u}_{h}^{k}\left(\overline{\boldsymbol{\psi}}_{h}^{k}\left(s ; t_{k+1}, \mathbf{x}\right)\right) \mathrm{d} s \\
& -\mathbf{P}\left(\boldsymbol{\zeta}_{h}^{k}\right) \mathbf{u}_{h}^{k}\left(\boldsymbol{\zeta}_{h}^{k}\right)\left(t-t_{k+1}\right), \\
\overline{\overline{\boldsymbol{\psi}}}_{h}^{k}\left(t ; t_{k+1}, \mathbf{x}\right)= & \mathbf{x}+\int_{t_{k+1}}^{t} \mathbf{U}_{h}^{k}\left(\overline{\overline{\boldsymbol{\psi}}}_{h}^{k}\left(s ; t_{k+1}, \mathbf{x}\right)\right) \mathrm{d} s .
\end{aligned}
$$

Subtracting the previous identities, we obtain

$$
\begin{aligned}
\overline{\boldsymbol{\psi}}_{h}^{k}\left(t ; t_{k+1}, \mathbf{x}\right)-\overline{\overline{\boldsymbol{\psi}}}_{h}^{k}\left(t ; t_{k+1}, \mathbf{x}\right) & =-\mathbf{u}_{h}^{k}\left(\boldsymbol{\zeta}_{h}^{k}\right) \Delta t-\mathbf{P}\left(\boldsymbol{\zeta}_{h}^{k}\right) \mathbf{u}_{h}^{k}\left(\boldsymbol{\zeta}_{h}^{k}\right)\left(t-t_{k+1}\right) \\
+ & \int_{t_{k+1}}^{t}\left(\mathbf{P}\left(\boldsymbol{\zeta}_{h}^{k}\right) \mathbf{u}_{h}^{k}\left(\overline{\boldsymbol{\psi}}_{h}^{k}\left(s ; t_{k+1}, \mathbf{x}\right)\right)-\mathbf{U}_{h}^{k}\left(\overline{\overline{\boldsymbol{\psi}}}_{h}^{k}\left(s ; t_{k+1}, \mathbf{x}\right)\right)\right) \mathrm{d} s
\end{aligned}
$$

then taking the $L^{2}(\mathcal{O})^{2}$-norm, we deduce that

$$
\begin{gathered}
\left\|\overline{\boldsymbol{\psi}}_{h}^{k}\left(t ; t_{k+1}, \cdot\right)-\overline{\overline{\boldsymbol{\psi}}}_{h}^{k}\left(t ; t_{k+1}, \cdot\right)\right\|_{L^{2}(\mathcal{O})^{2}} \leq C\left|\mathbf{u}_{h}^{k}\left(\boldsymbol{\zeta}_{h}^{k}\right)\right|\left(t-t_{k}\right)+C\left|\left(\mathbf{u}_{h}^{k}-\mathbf{P}\left(\boldsymbol{\zeta}_{h}^{k}\right) \mathbf{u}_{h}^{k}\right)\left(\boldsymbol{\zeta}_{h}^{k}\right)\right|\left(t_{k+1}-t\right) \\
+\int_{t}^{t_{k+1}}\left\|\mathbf{P}\left(\boldsymbol{\zeta}_{h}^{k}\right) \mathbf{u}_{h}^{k}\left(\overline{\boldsymbol{\psi}}_{h}^{k}\left(s ; t_{k+1}, \cdot\right)\right)-\mathbf{P}\left(\boldsymbol{\zeta}_{h}^{k}\right) \mathbf{U}_{h}^{k}\left(\overline{\boldsymbol{\psi}}_{h}^{k}\left(s ; t_{k+1}, \cdot\right)\right)\right\|_{L^{2}(\mathcal{O})^{2}} \mathrm{~d} s \\
\quad+\int_{t}^{t_{k+1}}\left\|\mathbf{P}\left(\boldsymbol{\zeta}_{h}^{k}\right) \mathbf{U}_{h}^{k}\left(\overline{\boldsymbol{\psi}}_{h}^{k}\left(s ; t_{k+1}, \cdot\right)\right)-\mathbf{U}_{h}^{k}\left(\overline{\boldsymbol{\psi}}_{h}^{k}\left(s ; t_{k+1}, \cdot\right)\right)\right\|_{L^{2}(\mathcal{O})^{2}} \mathrm{~d} s \\
\quad+\int_{t}^{t_{k+1}}\left\|\mathbf{U}_{h}^{k}\left(\overline{\boldsymbol{\psi}}_{h}^{k}\left(s ; t_{k+1}, \cdot\right)\right)-\mathbf{U}_{h}^{k}\left(\overline{\overline{\boldsymbol{\psi}}}_{h}^{k}\left(s ; t_{k+1}, \cdot\right)\right)\right\|_{L^{2}(\mathcal{O})^{2}} \mathrm{~d} s .
\end{gathered}
$$


By using 5.9 and the hypothesis 3.12 , the above estimate yields

$$
\begin{gathered}
\left\|\overline{\boldsymbol{\psi}}_{h}^{k}\left(t ; t_{k+1}, \cdot\right)-\overline{\overline{\boldsymbol{\psi}}}_{h}^{k}\left(t ; t_{k+1}, \cdot\right)\right\|_{L^{2}(\mathcal{O})^{2}} \leq C\left|\mathbf{u}_{h}^{k}\left(\boldsymbol{\zeta}_{h}^{k}\right)\right|\left(t-t_{k}\right)+C\left|\left(\mathbf{u}_{h}^{k}-\mathbf{P}\left(\boldsymbol{\zeta}_{h}^{k}\right) \mathbf{u}_{h}^{k}\right)\left(\boldsymbol{\zeta}_{h}^{k}\right)\right|\left(t_{k+1}-t\right) \\
+\left\|\mathbf{u}_{h}^{k}-\mathbf{U}_{h}^{k}\right\|_{L^{2}(\mathcal{O})^{2}}\left(t_{k+1}-t\right)+\left\|\mathbf{P}\left(\boldsymbol{\zeta}_{h}^{k}\right) \mathbf{U}_{h}^{k}-\mathbf{U}_{h}^{k}\right\|_{L^{2}(\mathcal{O})^{2}}\left(t_{k+1}-t\right) \\
+C \int_{t}^{t_{k+1}}\left\|\overline{\boldsymbol{\psi}}_{h}^{k}\left(s ; t_{k+1}, \cdot\right)-\overline{\overline{\boldsymbol{\psi}}}_{h}^{k}\left(s ; t_{k+1}, \cdot\right)\right\|_{L^{2}(\mathcal{O})^{2}} \mathrm{~d} s
\end{gathered}
$$

Then, applying the Gronwall inequality to the above estimate, for all $t \in\left[t_{k}, t_{k+1}\right]$ we deduce that

$$
\begin{gathered}
\left\|\overline{\boldsymbol{\psi}}_{h}^{k}\left(t ; t_{k+1}, \cdot\right)-\overline{\overline{\boldsymbol{\psi}}}_{h}^{k}\left(t ; t_{k+1}, \cdot\right)\right\|_{L^{2}(\mathcal{O})^{2}} \leq C\left|\mathbf{u}_{h}^{k}\left(\boldsymbol{\zeta}_{h}^{k}\right)\right|\left(t-t_{k}\right)+C\left|\left(\mathbf{u}_{h}^{k}-\mathbf{P}\left(\boldsymbol{\zeta}_{h}^{k}\right) \mathbf{u}_{h}^{k}\right)\left(\boldsymbol{\zeta}_{h}^{k}\right)\right|\left(t_{k+1}-t\right) \\
+\left\|\mathbf{u}_{h}^{k}-\mathbf{U}_{h}^{k}\right\|_{L^{2}(\mathcal{O})^{2}}\left(t_{k+1}-t\right)+\left\|\mathbf{P}\left(\boldsymbol{\zeta}_{h}^{k}\right) \mathbf{U}_{h}^{k}-\mathbf{U}_{h}^{k}\right\|_{L^{2}(\mathcal{O})^{2}}\left(t_{k+1}-t\right) \\
+C \Delta t^{2}\left(\left|\mathbf{u}_{h}^{k}\left(\boldsymbol{\zeta}_{h}^{k}\right)\right|+\left|\left(\mathbf{u}_{h}^{k}-\mathbf{P}\left(\boldsymbol{\zeta}_{h}^{k}\right) \mathbf{u}_{h}^{k}\right)\left(\boldsymbol{\zeta}_{h}^{k}\right)\right|\right. \\
\left.+\left\|\mathbf{u}_{h}^{k}-\mathbf{U}_{h}^{k}\right\|_{L^{2}(\mathcal{O})^{2}}+\left\|\mathbf{P}\left(\boldsymbol{\zeta}_{h}^{k}\right) \mathbf{U}_{h}^{k}-\mathbf{U}_{h}^{k}\right\|_{L^{2}(\mathcal{O})^{2}}\right)
\end{gathered}
$$

and in particular, taking $t=t_{k}$, we get

$$
\begin{aligned}
\left\|\overline{\mathbf{X}}_{h}^{k}-\overline{\overline{\mathbf{X}}}_{h}^{k}\right\|_{L^{2}(\mathcal{O})^{2}} \leq C \Delta t^{2}\left|\mathbf{u}_{h}^{k}\left(\boldsymbol{\zeta}_{h}^{k}\right)\right| & +C \Delta t\left(\left|\left(\mathbf{u}_{h}^{k}-\mathbf{P}\left(\boldsymbol{\zeta}_{h}^{k}\right) \mathbf{u}_{h}^{k}\right)\left(\boldsymbol{\zeta}_{h}^{k}\right)\right|\right. \\
& \left.+\left\|\mathbf{u}_{h}^{k}-\mathbf{U}_{h}^{k}\right\|_{L^{2}(\mathcal{O})^{2}}+\left\|\mathbf{P}\left(\boldsymbol{\zeta}_{h}^{k}\right) \mathbf{U}_{h}^{k}-\mathbf{U}_{h}^{k}\right\|_{L^{2}(\mathcal{O})^{2}}\right) \\
\leq C \Delta t^{2}\left\|\mathbf{U}_{h}^{k}\right\|_{L^{2}(\mathcal{O})^{2}} & +C \Delta t\left\|\mathbf{u}_{h}^{k}-\mathbf{U}_{h}^{k}\right\|_{L^{2}(\mathcal{O})^{2}}+C \Delta t\left\|\mathbf{P}\left(\boldsymbol{\zeta}_{h}^{k}\right) \mathbf{U}_{h}^{k}-\mathbf{U}_{h}^{k}\right\|_{L^{2}(\mathcal{O})^{2}}
\end{aligned}
$$

Combining the above inequality with the estimates 7.33 and 7.23 from Proposition 7.3 and using the hypothesis 3.12 , we conclude (7.28).

The proof of $(7.29)$ is completely similar to [18, eq. (7.6)].

Let us recall an approximation property of the projection on $K_{h}(\boldsymbol{\zeta}) \times M_{h}(\boldsymbol{\zeta})$ (see [18]).

Lemma 7.6. Suppose that $\mathbf{V} \in \mathcal{K}(\boldsymbol{\zeta})$ and that $P \in M(\boldsymbol{\zeta})$. Then there exists a unique couple $\left(\overline{\mathbf{V}}_{h}, \bar{P}_{h}\right)$ in $\mathcal{K}_{h}(\boldsymbol{\zeta}) \times M_{h}(\boldsymbol{\zeta})$ such that:

$$
\left\{\begin{array}{rll}
a\left(\mathbf{V}-\overline{\mathbf{V}}_{h}, \boldsymbol{\varphi}\right)+b\left(\boldsymbol{\varphi}, P-\bar{P}_{h}\right) & =0 & \forall \boldsymbol{\varphi} \in \mathcal{K}_{h}(\boldsymbol{\zeta}) \\
b\left(\mathbf{V}-\overline{\mathbf{V}}_{h}, q\right) & =0 & \forall q \in M_{h}(\boldsymbol{\zeta})
\end{array}\right.
$$

Moreover, if we suppose in addition that $\mathbf{V}_{\mid \mathcal{O} \backslash B(\boldsymbol{\zeta})} \in H^{2}(\mathcal{O} \backslash B(\boldsymbol{\zeta}))^{2}$ and that $P_{\mid \mathcal{O} \backslash B(\boldsymbol{\zeta})} \in$ $H^{1}(\mathcal{O} \backslash B(\boldsymbol{\zeta}))$, then there exists a positive constant $C$, independent of $h$, such that

$$
\left\|\mathbf{V}-\overline{\mathbf{V}}_{h}\right\|_{L^{2}(\mathcal{O})^{2}} \leq C h .
$$

\subsection{Error estimate}

In this section, we give the proof of our second main result stated in Theorem 4.1. First of all, let us observe that according to Lemma 7.6 there exists a unique couple $\left(\overline{\mathbf{U}}_{h}^{k+1}, \bar{P}_{h}^{k+1}\right) \in$ $\mathcal{K}_{h}\left(\boldsymbol{\zeta}_{h}^{k+1}\right) \times M_{h}\left(\boldsymbol{\zeta}_{h}^{k+1}\right)$ such that

$$
\left\{\begin{aligned}
a\left(\mathbf{U}_{h}^{k+1}-\overline{\mathbf{U}}_{h}^{k+1}, \boldsymbol{\varphi}\right)+b\left(\boldsymbol{\varphi}, P_{h}^{k+1}-\bar{P}_{h}^{k+1}\right) & =0 & \forall \boldsymbol{\varphi} \in \mathcal{K}_{h}\left(\boldsymbol{\zeta}_{h}^{k+1}\right), \\
b\left(\mathbf{U}_{h}^{k+1}-\overline{\mathbf{U}}_{h}^{k+1}, q\right) & =0 & \forall q \in M_{h}\left(\boldsymbol{\zeta}_{h}^{k+1}\right)
\end{aligned}\right.
$$

and moreover, there exists a positive constant $C$, independent of $h$ and $k$, such that the following estimate holds:

$$
\left\|\mathbf{U}_{h}^{k+1}-\overline{\mathbf{U}}_{h}^{k+1}\right\|_{L^{2}(\mathcal{O})^{2}} \leq C h .
$$


Subtracting the equations (4.7)- 4.8) from (7.4) $-(7.5)$ and using the definition of the projection $\left(\overline{\mathbf{U}}_{h}^{k+1}, \bar{P}_{h}^{k+1}\right)$ on the finite element spaces $\mathcal{K}_{h}\left(\boldsymbol{\zeta}_{h}^{k+1}\right) \times M_{h}\left(\boldsymbol{\zeta}_{h}^{k+1}\right)$ given in 7.35 , we get

$$
\begin{aligned}
\frac{1}{\Delta t}\left(\rho_{h}^{k+1}\left(\mathbf{U}_{h}^{k+1}-\mathbf{u}_{h}^{k+1}\right), \boldsymbol{\varphi}\right) & +a\left(\overline{\mathbf{U}}_{h}^{k+1}-\mathbf{u}_{h}^{k+1}, \boldsymbol{\varphi}\right)+b\left(\boldsymbol{\varphi}, \bar{P}_{h}^{k+1}-p_{h}^{k+1}\right) \\
= & \frac{1}{\Delta t}\left(\rho_{h}^{k+1} \widehat{\mathbf{J}}_{h}\left(\mathbf{U}_{h}^{k} \circ \widehat{\mathbf{X}}_{h}\right)-\rho_{h}^{k+1} \mathbf{u}_{h}^{k} \circ \overline{\mathbf{X}}_{h}^{k}, \boldsymbol{\varphi}\right) \\
& +\left(\left(\rho_{h}^{k+1}-\bar{\rho}_{h}^{k+1}\right) \mathbf{f}_{h}^{k+1}, \boldsymbol{\varphi}\right)+\left(\mathbf{A}_{h}^{k}, \boldsymbol{\varphi}\right) \quad \forall \boldsymbol{\varphi} \in \mathcal{K}_{h}\left(\boldsymbol{\zeta}_{h}^{k+1}\right), \\
b\left(\overline{\mathbf{U}}_{h}^{k+1}-\mathbf{u}_{h}^{k+1}, q\right)=0 \quad \forall q \in M_{h}\left(\boldsymbol{\zeta}_{h}^{k+1}\right) . &
\end{aligned}
$$

We choose the test functions

$$
\boldsymbol{\varphi}=\overline{\mathbf{U}}_{h}^{k+1}-\mathbf{u}_{h}^{k+1} \in \mathcal{K}_{h}\left(\boldsymbol{\zeta}_{h}^{k+1}\right) \quad \text { and } \quad q=\bar{P}_{h}^{k+1}-p_{h}^{k+1} \in M_{h}\left(\boldsymbol{\zeta}_{h}^{k+1}\right),
$$

then we obtain the following identity

$$
\begin{aligned}
\left(\rho_{h}^{k+1}\left(\mathbf{U}_{h}^{k+1}-\mathbf{u}_{h}^{k+1}\right), \overline{\mathbf{U}}_{h}^{k+1}-\mathbf{u}_{h}^{k+1}\right)+\Delta t a\left(\overline{\mathbf{U}}_{h}^{k+1}-\mathbf{u}_{h}^{k+1}, \overline{\mathbf{U}}_{h}^{k+1}-\mathbf{u}_{h}^{k+1}\right) & \\
= & \left(\rho_{h}^{k+1} \widehat{\mathbf{J}}_{h}\left(\mathbf{U}_{h}^{k} \circ \widehat{\mathbf{X}}_{h}\right)-\rho_{h}^{k+1} \mathbf{u}_{h}^{k} \circ \overline{\mathbf{X}}_{h}^{k}, \overline{\mathbf{U}}_{h}^{k+1}-\mathbf{u}_{h}^{k+1}\right) \\
& \quad+\Delta t\left(\left(\rho_{h}^{k+1}-\bar{\rho}_{h}^{k+1}\right) \mathbf{f}_{h}^{k+1}, \overline{\mathbf{U}}_{h}^{k+1}-\mathbf{u}_{h}^{k+1}\right)+\Delta t\left(\mathbf{A}_{h}^{k}, \overline{\mathbf{U}}_{h}^{k+1}-\mathbf{u}_{h}^{k+1}\right),
\end{aligned}
$$

which can be written as follows:

$$
\begin{aligned}
\left(\rho_{h}^{k+1}\left(\overline{\mathbf{U}}_{h}^{k+1}-\mathbf{u}_{h}^{k+1}\right), \overline{\mathbf{U}}_{h}^{k+1}-\mathbf{u}_{h}^{k+1}\right)+\Delta t a\left(\overline{\mathbf{U}}_{h}^{k+1}-\mathbf{u}_{h}^{k+1}, \overline{\mathbf{U}}_{h}^{k+1}-\mathbf{u}_{h}^{k+1}\right) \\
=\left(\rho_{h}^{k+1} \widehat{\mathbf{J}}_{h}\left(\mathbf{U}_{h}^{k} \circ \widehat{\mathbf{X}}_{h}\right)-\rho_{h}^{k+1} \mathbf{u}_{h}^{k} \circ \overline{\mathbf{X}}_{h}^{k}, \overline{\mathbf{U}}_{h}^{k+1}-\mathbf{u}_{h}^{k+1}\right) \\
+\left(\rho_{h}^{k+1}\left(\overline{\mathbf{U}}_{h}^{k+1}-\mathbf{U}_{h}^{k+1}\right), \overline{\mathbf{U}}_{h}^{k+1}-\mathbf{u}_{h}^{k+1}\right) \\
+\Delta t\left(\left(\rho_{h}^{k+1}-\bar{\rho}_{h}^{k+1}\right) \mathbf{f}_{h}^{k+1}, \overline{\mathbf{U}}_{h}^{k+1}-\mathbf{u}_{h}^{k+1}\right)+\Delta t\left(\mathbf{A}_{h}^{k}, \overline{\mathbf{U}}_{h}^{k+1}-\mathbf{u}_{h}^{k+1}\right) .
\end{aligned}
$$

By using the Cauchy-Schwarz inequality, there exists a positive constant $C$, independent of $h$ and $k$, such that

$$
\begin{aligned}
& \left\|\sqrt{\rho_{h}^{k+1}}\left(\overline{\mathbf{U}}_{h}^{k+1}-\mathbf{u}_{h}^{k+1}\right)\right\|_{L^{2}(\mathcal{O})^{2}} \leq\left\|\sqrt{\rho_{h}^{k+1}}\left(\widehat{\mathbf{J}}_{h}\left(\mathbf{U}_{h}^{k} \circ \widehat{\mathbf{X}}_{h}\right)-\mathbf{u}_{h}^{k} \circ \overline{\mathbf{X}}_{h}^{k}\right)\right\|_{L^{2}(\mathcal{O})^{2}} \\
& \quad+C\left(\left\|\overline{\mathbf{U}}_{h}^{k+1}-\mathbf{U}_{h}^{k+1}\right\|_{L^{2}(\mathcal{O})^{2}}+\Delta t\left\|\bar{\rho}_{h}^{k+1}-\rho_{h}^{k+1}\right\|_{L^{2}(\mathcal{O})}+\Delta t\left\|\mathbf{A}_{h}^{k}\right\|_{L^{2}(\mathcal{O})^{2}}\right) .
\end{aligned}
$$

Let us now estimate the first term in the right hand side of the inequality (7.37). To this end, we remark that since $\sqrt{\rho_{h}^{k+1}}=\sqrt{\rho_{h}^{k}} \circ \overline{\mathbf{X}}_{h}^{k}$ (see (5.8)), one can write

$$
\begin{aligned}
\sqrt{\rho_{h}^{k+1}}\left(\widehat{\mathbf{J}}_{h}\left(\mathbf{U}_{h}^{k} \circ \widehat{\mathbf{X}}_{h}\right)-\mathbf{u}_{h}^{k} \circ \overline{\mathbf{X}}_{h}^{k}\right)= & \sqrt{\rho_{h}^{k+1}}\left(\widehat{\mathbf{J}}_{h}-\mathbf{I} \mathbf{d}\right) \mathbf{U}_{h}^{k} \circ \widehat{\mathbf{X}}_{h} \\
& +\sqrt{\rho_{h}^{k+1}}\left(\mathbf{U}_{h}^{k} \circ \widehat{\mathbf{X}}_{h}-\mathbf{U}_{h}^{k} \circ \overline{\mathbf{X}}_{h}^{k}\right) \\
& +\sqrt{\rho_{h}^{k+1}}\left(\mathbf{U}_{h}^{k}-\overline{\mathbf{U}}_{h}^{k}\right) \circ \overline{\mathbf{X}}_{h}^{k} \\
& +\left(\sqrt{\rho_{h}^{k}}\left(\overline{\mathbf{U}}_{h}^{k}-\mathbf{u}_{h}^{k}\right)\right) \circ \overline{\mathbf{X}}_{h}^{k} .
\end{aligned}
$$

Then, by using the hypothesis 3.12 and the fact that $\operatorname{det} \mathbf{J}_{\overline{\mathbf{X}}_{h}^{k}}=1$, we deduce the following estimate:

$$
\begin{aligned}
\left\|\sqrt{\rho_{h}^{k+1}}\left(\widehat{\mathbf{J}}_{h}\left(\mathbf{U}_{h}^{k} \circ \widehat{\mathbf{X}}_{h}\right)-\mathbf{u}_{h}^{k} \circ \overline{\mathbf{X}}_{h}^{k}\right)\right\|_{L^{2}(\mathcal{O})^{2}} \\
\leq C\left\|\widehat{\mathbf{J}}_{h}-\mathbf{I d}\right\|_{L^{2}(\mathcal{O})^{4}}+C\left\|\widehat{\mathbf{X}}_{h}-\overline{\mathbf{X}}_{h}^{k}\right\|_{L^{2}(\mathcal{O})^{2}} \\
\quad+C\left\|\overline{\mathbf{U}}_{h}^{k}-\mathbf{U}_{h}^{k}\right\|_{L^{2}(\mathcal{O})^{2}}+\left\|\sqrt{\rho_{h}^{k}}\left(\overline{\mathbf{U}}_{h}^{k}-\mathbf{u}_{h}^{k}\right)\right\|_{L^{2}(\mathcal{O})^{2}}
\end{aligned}
$$


Combining the estimates 7.37 together with (7.38), we deduce that

$$
\begin{aligned}
\left\|\sqrt{\rho_{h}^{k+1}}\left(\overline{\mathbf{U}}_{h}^{k+1}-\mathbf{u}_{h}^{k+1}\right)\right\|_{L^{2}(\mathcal{O})^{2}} \leq\left\|\sqrt{\rho_{h}^{k}}\left(\overline{\mathbf{U}}_{h}^{k}-\mathbf{u}_{h}^{k}\right)\right\|_{L^{2}(\mathcal{O})^{2}} \\
+C\left(\left\|\widehat{\mathbf{J}}_{h}-\mathbf{I d}\right\|_{L^{2}(\mathcal{O})^{4}}+\left\|\widehat{\mathbf{X}}_{h}-\overline{\mathbf{X}}_{h}^{k}\right\|_{L^{2}(\mathcal{O})^{2}}+\left\|\overline{\mathbf{U}}_{h}^{k}-\mathbf{U}_{h}^{k}\right\|_{L^{2}(\mathcal{O})^{2}}\right. \\
\left.\quad+\left\|\overline{\mathbf{U}}_{h}^{k+1}-\mathbf{U}_{h}^{k+1}\right\|_{L^{2}(\mathcal{O})^{2}}+\Delta t\left\|\bar{\rho}_{h}^{k+1}-\rho_{h}^{k+1}\right\|_{L^{2}(\mathcal{O})}+\Delta t\left\|\mathbf{A}_{h}^{k}\right\|_{L^{2}(\mathcal{O})^{2}}\right) .
\end{aligned}
$$

Due to Lemma 7.5 (see $7.28-77.29)$ and Lemma 7.4 the above estimate yields

$$
\begin{aligned}
\| \sqrt{\rho_{h}^{k+1}}\left(\overline{\mathbf{U}}_{h}^{k+1}-\mathbf{u}_{h}^{k+1}\right) & \left\|_{L^{2}(\mathcal{O})^{2}} \leq\right\| \sqrt{\rho_{h}^{k}}\left(\overline{\mathbf{U}}_{h}^{k}-\mathbf{u}_{h}^{k}\right) \|_{L^{2}(\mathcal{O})^{2}}+C\left(\Delta t\left|\boldsymbol{\zeta}\left(t_{k}\right)-\boldsymbol{\zeta}_{h}^{k}\right|+\Delta t\left\|\mathbf{U}_{h}^{k}-\mathbf{u}_{h}^{k}\right\|_{L^{2}(\mathcal{O})^{2}}\right. \\
& +\sqrt{\Delta t}\left\|\boldsymbol{\delta}_{k}\right\|_{L^{2}\left(\mathcal{O} \times\left(t_{k}, t_{k+1}\right)\right)^{2}}+\Delta t^{2}+\Delta t \sqrt{h} \\
& \left.+\left\|\overline{\mathbf{U}}_{h}^{k}-\mathbf{U}_{h}^{k}\right\|_{L^{2}(\mathcal{O})^{2}}+\left\|\overline{\mathbf{U}}_{h}^{k+1}-\mathbf{U}_{h}^{k+1}\right\|_{L^{2}(\mathcal{O})^{2}}+\Delta t\left\|\mathbf{A}_{h}^{k}\right\|_{L^{2}(\mathcal{O})^{2}}\right) .
\end{aligned}
$$

Let us now use the estimate 7.36 of the projection, the estimates 7.6 and 7.30 of $\mathbf{A}_{h}^{k}$, respectively $\boldsymbol{\delta}_{k}$, then the above inequality becomes

$$
\begin{aligned}
& \left\|\sqrt{\rho_{h}^{k+1}}\left(\overline{\mathbf{U}}_{h}^{k+1}-\mathbf{u}_{h}^{k+1}\right)\right\|_{L^{2}(\mathcal{O})^{2}} \leq(1+C \Delta t)\left\|\sqrt{\rho_{h}^{k}}\left(\overline{\mathbf{U}}_{h}^{k}-\mathbf{u}_{h}^{k}\right)\right\|_{L^{2}(\mathcal{O})^{2}} \\
& +C\left(\Delta t^{2}+\Delta t \sqrt{h}+h+\Delta t\left|\boldsymbol{\zeta}\left(t_{k}\right)-\boldsymbol{\zeta}_{h}^{k}\right|+\Delta t\left|\boldsymbol{\zeta}\left(t_{k+1}\right)-\boldsymbol{\zeta}_{h}^{k+1}\right|\right. \\
& \left.\quad+(\Delta t)^{3 / 2}\left\|\frac{\partial \mathbf{u}}{\partial t}\right\|_{L^{2}\left(\mathcal{O} \times\left(t_{k}, t_{k+1}\right)\right)^{2}}+(\Delta t)^{3 / 2}\left\|\frac{d^{2}}{d t^{2}}[\mathbf{u} \circ \widetilde{\boldsymbol{\psi}}]\right\|_{L^{2}\left(\mathcal{O} \times\left(t_{k}, t_{k+1}\right)\right)^{2}}\right) .
\end{aligned}
$$

We also recall (see [18, Eq.(7.9)]) that

$$
\left|\boldsymbol{\zeta}\left(t_{k+1}\right)-\boldsymbol{\zeta}_{h}^{k+1}\right| \leq\left|\boldsymbol{\zeta}\left(t_{k}\right)-\boldsymbol{\zeta}_{h}^{k}\right|+C \Delta t|| \mathbf{U}_{h}^{k}-\mathbf{u}_{h}^{k} \|_{L^{2}(\mathcal{O})^{2}}+C \Delta t^{2},
$$

then since the estimate of the projection 7.36 holds, we get

$$
\left|\boldsymbol{\zeta}\left(t_{k+1}\right)-\boldsymbol{\zeta}_{h}^{k+1}\right| \leq\left|\boldsymbol{\zeta}\left(t_{k}\right)-\boldsymbol{\zeta}_{h}^{k}\right|+C \Delta t\left\|\sqrt{\rho_{h}^{k}}\left(\overline{\mathbf{U}}_{h}^{k}-\mathbf{u}_{h}^{k}\right)\right\|_{L^{2}(\mathcal{O})^{2}}+C h \Delta t+C \Delta t^{2} .
$$

Therefore, taking into account the inequality $(7.40)$ in the estimate (7.39), one can deduce that

$$
\begin{aligned}
&\left\|\sqrt{\rho_{h}^{k+1}}\left(\overline{\mathbf{U}}_{h}^{k+1}-\mathbf{u}_{h}^{k+1}\right)\right\|_{L^{2}(\mathcal{O})^{2}}+\left|\boldsymbol{\zeta}\left(t_{k+1}\right)-\boldsymbol{\zeta}_{h}^{k+1}\right| \\
& \leq(1+C \Delta t)\left(\left\|\sqrt{\rho_{h}^{k}}\left(\overline{\mathbf{U}}_{h}^{k}-\mathbf{u}_{h}^{k}\right)\right\|_{L^{2}(\mathcal{O})^{2}}+\left|\boldsymbol{\zeta}\left(t_{k}\right)-\boldsymbol{\zeta}_{h}^{k}\right|\right) \\
&+C\left(\Delta t^{2}+\Delta t \sqrt{h}+h+(\Delta t)^{3 / 2}\left\|\frac{\partial \mathbf{u}}{\partial t}\right\|_{L^{2}\left(\mathcal{O} \times\left(t_{k}, t_{k+1}\right)\right)^{2}}\right. \\
&\left.+(\Delta t)^{3 / 2}\left\|\frac{d^{2}}{d t^{2}}[\mathbf{u} \circ \widetilde{\boldsymbol{\psi}}]\right\|_{L^{2}\left(\mathcal{O} \times\left(t_{k}, t_{k+1}\right)\right)^{2}}\right) .
\end{aligned}
$$

By applying the discrete Gronwall Lemma in (7.41, there exists a positive constant independent of $h$ such that

$$
\begin{aligned}
\left\|\sqrt{\rho_{h}^{k}}\left(\overline{\mathbf{U}}_{h}^{k}-\mathbf{u}_{h}^{k}\right)\right\|_{L^{2}(\mathcal{O})^{2}}+\left|\boldsymbol{\zeta}\left(t_{k}\right)-\boldsymbol{\zeta}_{h}^{k}\right| & \\
\leq C_{1}\left(\Delta t+\sqrt{h}+\frac{h}{\Delta t}+\right. & (\Delta t)^{3 / 2}\left\|\frac{\partial \mathbf{u}}{\partial t}\right\|_{L^{2}(\mathcal{O} \times(0, T))^{2}} \\
& \left.+(\Delta t)^{3 / 2}\left\|\frac{d^{2}}{d t^{2}}[\mathbf{u} \circ \widetilde{\boldsymbol{\psi}}]\right\|_{L^{2}(\mathcal{O} \times(0, T))^{2}}\right),
\end{aligned}
$$


for all $k \in\{0, \ldots, N\}$.

Using the hypothesis (3.12), the estimate of the projection (7.36), Lemma 5.6 in [18, and the fact that $\sqrt{\rho_{h}^{k}} \geq \min \left\{\sqrt{\rho_{f}}, \sqrt{\rho_{s}}\right\}>0$, we get

$$
\left\|\mathbf{u}\left(t_{k}\right)-\mathbf{u}_{h}^{k}\right\|_{L^{2}(\mathcal{O})^{2}}+\left|\boldsymbol{\zeta}\left(t_{k}\right)-\boldsymbol{\zeta}_{h}^{k}\right| \leq C\left(\Delta t+\sqrt{h}+\frac{h}{\Delta t}\right) \quad \forall k \in\{0, \ldots, N\} .
$$

Then, for $\Delta t$ small enough and $h \leq C_{0} \Delta t^{1+\alpha}$ with $0<\alpha \leq 1$, we conclude that the estimate (7.43) becomes

$$
\left\|\mathbf{u}\left(t_{k}\right)-\mathbf{u}_{h}^{k}\right\|_{L^{2}(\mathcal{O})^{2}}+\left|\boldsymbol{\zeta}\left(t_{k}\right)-\boldsymbol{\zeta}_{h}^{k}\right| \leq C \Delta t^{\alpha} \quad \forall k \in\{0, \ldots, N\},
$$

which is the conclusion of Theorem 4.1 .

\section{Acknowledgements}

J. San Martín was partially supported by Grant Fondecyt 1090239 and BASAL-CMM Project. J.-F. Scheid gratefully acknowledges the Program ECOS-CONICYT (Scientific cooperation project between France and Chile) through grant C07-E05. L. Smaranda was partially supported by Grant RP-2, no. 6/01.07.2009 of CNCSIS-UEFISCSU.

\section{References}

[1] Y. Achdou And J.-L. Guermond, Convergence analysis of a finite element projection/Lagrange-Galerkin method for the incompressible Navier-Stokes equations, SIAM J. Numer. Anal., 37 (2000), pp. 799-826 (electronic).

[2] V. ARnold, Ordinary differential equations, Springer-Verlag, Berlin, 1992. Translated from the third Russian edition by Roger Cooke.

[3] R. J. DiPerna And P.-L. Lions, Ordinary differential equations, transport theory and Sobolev spaces, Invent. Math., 98 (1989), pp. 511-547.

[4] L. Formaggia and F. Nobile, A stability analysis for the arbitrary Lagrangian Eulerian formulation with finite elements, East-West J. Numer. Math., 7 (1999), pp. 105131.

[5] L. Gastaldi, A priori error estimates for the arbitrary Lagrangian Eulerian formulation with finite elements, East-West J. Numer. Math., 9 (2001), pp. 123-156.

[6] V. Girault and P.-A. Raviart, Finite element approximation of the Navier-Stokes equations, Springer-Verlag, Berlin, 1979.

[7] R. Glowinski, T.-W. Pan, T. Hesla, D. Joseph, and J. Périaux, A distributed Lagrange multiplier/fictitious domain method for the simulation of flow around moving rigid bodies: application to particulate flow, Comput. Methods Appl. Mech. Engrg., 184 (2000), pp. 241-267. Vistas in domain decomposition and parallel processing in computational mechanics.

[8] R. Glowinski, T.-W. Pan, T. I. Hesla, D. D. Joseph, and J. Périaux, A fictitious domain approach to the direct numerical simulation of incompressible viscous flow past moving rigid bodies: application to particulate flow, J. Comput. Phys., 169 (2001), pp. 363-426.

[9] C. Grandmont, V. Guimet, and Y. Maday, Numerical analysis of some decoupling techniques for the approximation of the unsteady fluid structure interaction, Math. Models Methods Appl. Sci., 11 (2001), pp. 1349-1377.

[10] G. Legendre and T. TAKAhashi, Convergence of a Lagrange-Galerkin method for a fluid-rigid body system in ALE formulation, M2AN Math. Model. Numer. Anal., 42 (2008), pp. 609-644. 
[11] B. Maury, Direct simulations of $2 D$ fluid-particle flows in biperiodic domains, J. Comput. Phys., 156 (1999), pp. 325-351.

[12] B. Maury and R. Glowinski, Fluid-particle flow: a symmetric formulation, C. R. Acad. Sci. Paris Sér. I Math., 324 (1997), pp. 1079-1084.

[13] S. Osher And J. A. Sethian, Fronts propagating with curvature-dependent speed: algorithms based on Hamilton-Jacobi formulations, J. Comput. Phys., 79 (1988), pp. 1249.

[14] C. S. Peskin, The immersed boundary method, Acta Numer., 11 (2002), pp. 479-517.

[15] O. Pironneau, On the transport-diffusion algorithm and its applications to the NavierStokes equations, Numer. Math., 38 (1982), pp. 309-332.

[16] A. Quarteroni And A. Valli, Numerical approximation of partial differential equations, vol. 23 of Springer Series in Computational Mathematics, Springer-Verlag, Berlin, 1994.

[17] J. San Martín, J.-F. Scheid, T. Takahashi, and M. Tucsnak, Convergence of the Lagrange-Galerkin method for a fluid-rigid system, C. R. Math. Acad. Sci. Paris, 339 (2004), pp. 59-64.

[18] — Convergence of the Lagrange-Galerkin method for the equations modelling the motion of a fluid-rigid system, SIAM J. Numer. Anal., 43 (2005), pp. 1536-1571 (electronic).

[19] — An initial and boundary value problem modeling of fish-like swimming, Arch. Ration. Mech. Anal., 188 (2008), pp. 429-455.

[20] J. San Martín, L. Smaranda, and T. Takahashi, Convergence of a finite element/ALE method for the Stokes equations in a domain depending on time, J. Comput. Appl. Math., 230 (2009), pp. 521-545.

[21] E. SüLI, Convergence and nonlinear stability of the Lagrange-Galerkin method for the Navier-Stokes equations, Numer. Math., 53 (1988), pp. 459-483.

[22] R. Temam, Problèmes mathématiques en plasticité, Gauthier-Villars, Montrouge, 1983. 